\title{
Longtime behavior of a diffuse interface model for binary fluid mixtures with shear dependent viscosity
}

\author{
MAURIZIO GRASSELLI \\ Dipartimento di Matematica "F. Brioschi", Politecnico di Milano, \\ Via E. Bonardi 9 I-20133 Milano, Italy \\ E-mail:maurizio.grasselli@polimi.it \\ DALIBOR PRAŽÁK \\ Department of Mathematical Analysis, Charles University, \\ Sokolovská 83, CZ-18675 Prague 8, Czech Republic \\ E-mail:prazak@karlin.mff.cuni.cz.
}

[Received 29 September 2010 and in revised form 7 October 2011]

In memoriam Andrea Maffio (1962-2009)

\begin{abstract}
We consider a system which describes the behavior of a binary mixture of immiscible incompressible fluids with shear dependent viscosity by means of the diffuse interface approach. This system consists of Navier-Stokes type equations, characterized by a nonlinear stress-strain law, which are nonlinearly coupled with a convective Cahn-Hilliard equation for the order parameter. We analyze the corresponding dynamical system and, by means of the short trajectory method, we prove the existence of global and exponential attractors. We also discuss the dependence of an upper bound of the fractal dimension on the physical parameters of the system.
\end{abstract}

2010 Mathematics Subject Classification: 35B41, 35K55, 35Q35, 76A05, 76D05, 76T99.

Keywords: Incompressible viscous fluids; shear dependent viscosity; binary mixtures; diffuse interfaces; Cahn-Hilliard equations; global attractors; exponential attractors; fractal dimension.

\section{Introduction}

The mathematical treatment of sharp interface problems is rather complicated also from the numerical viewpoint. Therefore, it is particularly convenient to introduce models where the interface is diffused (i.e., it has some small thickness $\varepsilon$ ). This is done by means of a convenient order parameter whose evolution is governed by a gradient flow type equation (see, e.g., [3]). For instance, if we want to describe the motion of a (homogeneous) incompressible, isothermal and immiscible binary fluid mixture (e.g., oil and water), a typical model is the so-called Cahn-HilliardNavier-Stokes system for the (mean) fluid velocity $\mathbf{u}$ and the order parameter $\phi$ (i.e., the relative concentration of one phase). This system reads as follows (with unit density):

$$
\begin{aligned}
& \partial_{t} \mathbf{u}+(\mathbf{u} \cdot \nabla) \mathbf{u}-\nabla \cdot \boldsymbol{\tau}(\phi, \mathbf{e}(\mathbf{u}))+\nabla \pi=k \mu \nabla \phi+\mathbf{g}, \\
& \nabla \cdot \mathbf{u}=\mathbf{0}, \\
& \partial_{t} \phi+\mathbf{u} \cdot \nabla \phi-m \Delta \mu=0, \\
& \mu=-\varepsilon \Delta \phi+\alpha F^{\prime}(\phi),
\end{aligned}
$$


in $\Omega \times(0, T), \Omega \subseteq \mathbb{R}^{N}, N=2,3, T>0$. Here $k, \varepsilon, m, \alpha$ are given positive constants, $\pi$ denotes the pressure, $\mathbf{g}$ is a given (time-independent) external force and $F$ is a given double-well potential. The stress tensor $\tau$ is defined by the constitutive relation

$$
\tau(\phi, \mathbf{e}(\mathbf{u}))=v(\phi) \mathbf{e}(\mathbf{u})
$$

where $v$ is a strictly positive function and $\mathbf{e}$ is the symmetric velocity gradient, namely,

$$
\mathbf{e}(\mathbf{u}):=\frac{1}{2}\left(\nabla \mathbf{u}+(\nabla \mathbf{u})^{t r}\right)
$$

System (1.1)-(1.5) is known as model $\mathrm{H}$ and was proposed in [19] (see also [29]) and then rigorously justified in [15]. From the mathematical viewpoint, this system has been first studied in [31] for $\Omega=\mathbb{R}^{2}$. Then, in the case of bounded domains, a careful analysis has been carried out in [6] (see also [7]). More recently, the case of logarithmic potentials and constant mobility $m$ has been considered in [1], where, in particular, the convergence of solutions to a single equilibrium has been established in absence of nongradient external forces. This issue has also been investigated in [33] for smooth potentials. A rather complete picture of the longtime behavior in the case $N=2$ on a bounded domain can be found in [13]. In the case $N=3$, existence of trajectory attractors has been demonstrated in [14] with time-dependent external forces (see also [2] for an alternative approach in the case $\mathbf{g} \equiv \mathbf{0}$ ). Regarding the numerical analysis of Cahn-Hilliard-Navier-Stokes systems we refer the reader to, e.g., $[4,12,16,17,21,28]$ and their references.

Here we want to consider a nontrivial generalization of this model which accounts for a shear dependent viscosity. More precisely, instead of (1.5), we assume the following stress-strain relationship:

$$
\tau(\phi, \mathbf{e}(\mathbf{u}))=\left(v_{1}(\phi)+v_{2}(\phi)|\mathbf{e}(\mathbf{u})|^{p-2}\right) \mathbf{e}(\mathbf{u}) .
$$

Here $v_{i}$ are strictly positive given functions and $p>1$. Of course, in the case $p=2$ we obtain the previous model.

In the case of single fluids, assumption (1.7) is known as the Ladyzhenskaya model (see [20]), while the particular case $p=3$ is the Smagorinsky model of turbulence (cf. [30]). We recall that, in the case $N=3$, the main features of the corresponding generalized Navier-Stokes equations endowed with, say, no-slip or periodic boundary conditions are the uniqueness of weak solutions if $p \geqslant 5 / 2$ and the existence of a (unique) strong solution if $p \geqslant 11 / 5$. This problem has been widely and deeply investigated in recent years by several people (see [5, 11, 23, 25] and references therein). Regarding the asymptotic behavior, in the seminal paper [22], the authors introduced a new method to prove the existence of a global attractor of finite fractal dimension. This approach, now known as the short trajectory method, has been refined in [24] (see also [11, 7.4]) to prove the existence of exponential attractors as well. This method is actually rather flexible and it has been applied to many other dynamical systems so far (see, e.g., [26, Rem. 3.8] and references therein).

System (1.1)-(1.4) with a generalized version of (1.7) has been considered first in [18]. There, some existence, uniqueness and regularity results have been proven on bounded domains and for periodic or no-slip and no-flux boundary conditions for $\mathbf{u}$ and $\phi, \mu$, respectively. The case of singular potentials has also been considered, proving the existence of a measure-valued solution. Here, in the case of smooth potentials and periodic boundary conditions, we want to investigate the asymptotic behavior of (1.1)-(1.4), (1.7) along the lines of [24]. More precisely, using the short trajectory method, we prove the existence of a global attractor and of an exponential attractor. In 
addition, we observe that the upper bound of the fractal dimension of these attractors grows at most polynomially with the data. We will essentially focus on the case $N=3$ with $p \geqslant 11 / 5$. The degenerate case $p \in(1,2)$ and $N=2$ will be treated elsewhere. Boundary conditions like no-slip for $\mathbf{u}$ and no-flux for $\phi$ and $\mu$ might also be considered possibly with further restrictions, e.g., on $p$ (see $[11,7.4]$ and references therein for simple fluids, cf. also Remark 2.7).

The present analysis requires combining some different existing, albeit nontrivial, techniques and we think that it is a further significant application of the short trajectory approach. Moreover, this is a first step towards the possibility of considering similar models with more challenging features like, say, singular potentials and degenerate mobility coefficients.

Equations (1.1)-(1.4) and (1.7) are given in $\mathbb{R}^{N} \times(0, \infty)$, and $\mathbf{u}, \phi$ and the chemical potential $\mu$ are supposed to be $L$-periodic with respect to each variable $x_{k}, k=1, \ldots, N$. The system is also endowed with initial conditions

$$
\mathbf{u}(0)=\mathbf{u}_{0}, \quad \phi(0)=\phi_{0} .
$$

The plan of the paper is the following. In Section 2 we introduce the basic assumptions and we define the notion of weak solution. Then we state and prove some existence, regularity and continuous dependence results. Section 3 will be devoted to the main results about the asymptotic behavior, that is, the existence of global and exponential attractors.

\section{Well-posedness and smoothness}

The aim of this section is to establish main results concerning the properties of solutions to our system. Namely, we prove the compactness of the set of weak solutions (Theorem 2.4), which is tantamount to the (global) existence of weak solutions. A key ingredient of the subsequent analysis is the regularity result (Theorem 2.6), which ensures the uniqueness of solutions (Theorem 2.8) under sufficiently general conditions.

Let us begin by stating our assumptions on the potential $F$ :

$$
\begin{aligned}
& F \in C^{3}(\mathbb{R} ; \mathbb{R}), \\
& \liminf _{|y| \rightarrow \infty} F^{\prime \prime}(y)>0, \\
& \left|F^{(3)}(y)\right| \leqslant C_{F}\left(1+|y|^{r-1}\right), \quad r \in \begin{cases}{[1,3],} & N=3, \\
{[1, \infty),} & N=2 .\end{cases}
\end{aligned}
$$

Moreover, for the sake of simplicity, we can assume $F \geqslant 0$.

Regarding $v_{i}, i=1,2$, we suppose

$$
\begin{aligned}
& v_{1}, \nu_{2} \in C^{1}(\mathbb{R} ; \mathbb{R}), \\
& \min _{y \in \mathbb{R}} v_{i}(y) \geqslant v_{*}^{i}, \quad \max _{y \in \mathbb{R}} v_{i}(y) \leqslant v_{i}^{*}, \quad \max _{y \in \mathbb{R}}\left|v_{i}^{\prime}(y)\right| \leqslant v_{i}^{\sharp},
\end{aligned}
$$

for some positive $v_{*}^{i}, v_{i}^{*}$, and $v_{i}^{\sharp}$. 
Also, we take $m=1$ in (1.3) and we observe that the stress tensor $\tau$ has the following properties:

$$
\begin{aligned}
& \tau(\phi, \mathbf{0})=\mathbf{0}, \\
& |\tau(\phi, \mathbf{e})-\tau(\phi, \tilde{\mathbf{e}})| \leqslant c_{1} v^{*}(1+|\mathbf{e}|+|\tilde{\mathbf{e}}|)^{p-2}|\mathbf{e}-\tilde{\mathbf{e}}|, \\
& |\tau(\phi, \mathbf{e})-\tau(\tilde{\phi}, \mathbf{e})| \leqslant c_{2} v^{\sharp}(1+|\mathbf{e}|)^{p-1}|\phi-\tilde{\phi}|, \\
& (\tau(\phi, \mathbf{e})-\tau(\phi, \tilde{\mathbf{e}})):(\mathbf{e}-\tilde{\mathbf{e}}) \geqslant c_{3} v_{*}(1+|\mathbf{e}|+|\tilde{\mathbf{e}}|)^{p-2}|\mathbf{e}-\tilde{\mathbf{e}}|^{2},
\end{aligned}
$$

where

$$
v^{*}:=\max \left\{v_{1}^{*}, v_{2}^{*}\right\}, \quad v_{*}:=\min \left\{v_{*}^{1}, v_{*}^{2}\right\}, \quad v^{\sharp}:=\max \left\{v_{1}^{\sharp}, v_{2}^{\sharp}\right\},
$$

for some suitable $p$ such that

$$
p \geqslant \frac{3 N+2}{N+2}
$$

All the functions here considered are $L$-periodic in space with respect to each variable and the reference domain is $\Omega=(0, L)^{N}$. By $H_{\text {per }}^{a}(\Omega), a>0$, we denote the Banach space of $L$-periodic functions which belong to $W^{a, 2}(\Omega)$ whose norm is defined by $\|\cdot\|_{H_{p e r}^{a}(\Omega)}=\|\cdot\|_{W^{a, 2}(\Omega)}$. The notation $L_{d i v}^{2}$ and $W_{d i v}^{a, p}, a>0$, is reserved for the vector-valued functions of $L^{2}\left(\Omega ; \mathbb{R}^{N}\right)$ and $W^{a, p}\left(\Omega ; \mathbb{R}^{N}\right)$ which are divergence-free and have zero mean value. The latter canonical norms are indicated as $\|\cdot\|_{2}$ and $\|\cdot\|_{a, p}$, respectively. An equivalent norm $\|\nabla \cdot\|_{p}$ is often used as well. In addition, if $V$ is a Banach space, then $V^{*}$ stands for its dual.

DEFinition 2.1 Assume $\left(\mathbf{u}_{0}, \phi_{0}\right) \in L_{d i v}^{2} \times H_{p e r}^{1}$ and $\mathbf{g} \in\left(W_{d i v}^{1,2}\right)^{*}$. For any given $T>0$, the pair $(\mathbf{u}, \phi)$ is called a weak solution provided that

$$
\begin{aligned}
& \mathbf{u} \in L^{\infty}\left(0, T ; L_{d i v}^{2}\right) \cap L^{p}\left(0, T ; W_{d i v}^{1, p}\right), \\
& \phi \in L^{\infty}\left(0, T ; H_{p e r}^{1}\right) \cap L^{2}\left(0, T ; H_{p e r}^{3}\right),
\end{aligned}
$$

and (1.1)-(1.4) hold in the sense of distributions in $\Omega \times(0, T)$ with (1.7) and (1.8). Concerning (1.1), only test functions with zero (spatial) divergence are considered. In addition, the spatial average of $\phi$ is conserved, that is,

$$
\bar{\phi}(t) \equiv \bar{\phi}_{0}, \quad \forall t \geqslant 0,
$$

where $\bar{\phi}:=|\Omega|^{-1} \int_{\Omega} \phi \mathrm{d} x$.

REMARK 2.2 Note that (2.8) follows by taking 1 as test function in (1.3). Moreover, due to our assumptions (2.1) and (2.5) all the nonlinearities are integrable, and for finite $T>0$,

$$
\begin{aligned}
& \partial_{t} \mathbf{u} \in L^{p^{\prime}}\left(0, T ;\left(W_{d i v}^{1, p}\right)^{*}\right), \\
& \partial_{t} \phi \in L^{2}\left(0, T ;\left(H_{p e r}^{1}\right)^{*}\right) .
\end{aligned}
$$

Consequently, any weak solution has a representative

$$
(\mathbf{u}, \phi) \in C\left([0, T) ; L_{d i v}^{2} \times H_{p e r}^{1}\right),
$$

so that (1.8) makes sense. Note that $\mathbf{u}, \phi$ and $\Delta \phi$ are admissible test functions for (1.1) and (1.3), respectively. Finally, we note that the pressure $\pi$ is excluded from the subsequent analysis thanks to the fact we only work with divergence free test functions for (1.1). In the current setting of periodic boundary conditions, the existence of a function $\pi$ such that (1.1) holds in the sense of distributions can be established a posteriori (see, e.g., [32, Ch. 3, Prop. 1.1]). 
REMARK 2.3 We will observe throughout our analysis that, as one might expect, the most difficult term to handle is the convective one in (1.1), which gives rise to the lower bound (2.5) (see [23, Ch. 5, Lemma 2.44]).

For future reference, it will be useful to write down the abstract weak formulation of (1.1) explicitly, that is,

$$
\left\langle\partial_{t} \mathbf{u}+N(\phi, \mathbf{e}(\mathbf{u}))+\mathbf{u} \cdot \nabla \mathbf{u}-k \mu \nabla \phi-\mathbf{g}, \mathbf{v}\right\rangle=0, \quad \forall \mathbf{v} \in L^{p}\left(0, T ; W_{d i v}^{1, p}\right),
$$

where $\langle\cdot, \cdot\rangle$ stands for the duality between $L^{p^{\prime}}\left(0, T ;\left(W_{d i v}^{1, p}\right)^{*}\right)$ and $L^{p}\left(0, T ; W_{d i v}^{1, p}\right)$, and

$$
\langle N(\phi, \mathbf{e}(\mathbf{u})), \mathbf{v}\rangle=\int_{\Omega \times(0, T)} \tau(\phi, \mathbf{e}(\mathbf{u})): \mathbf{e}(\mathbf{v}) \mathrm{d} x \mathrm{~d} t .
$$

THEOREM 2.4 Let $\left(\mathbf{u}^{n}, \phi^{n}\right)$ be a sequence of weak solutions such that $\left(\mathbf{u}^{n}(0), \phi^{n}(0)\right) \rightarrow\left(\mathbf{u}_{0}, \phi_{0}\right)$ in $L_{\text {div }}^{2} \times H_{\text {per }}^{1}$. Then, modulo a subsequence, $\mathbf{u}^{n} \rightarrow \mathbf{u}, \phi^{n} \rightarrow \phi$ in the spaces specified in (2.17) and (2.18) below, and $(\mathbf{u}, \phi)$ is again a weak solution.

Proof. We confine ourselves to the case $N=3$. The two-dimensional case can be treated in the same way with a more general growth condition on $F$ (cf. (2.1)). Also, from now on, $C_{F}$ will denote a positive constant which controls the growth of $F$ and depends on $F$ only, but it may change from line to line. Multiply (1.1) by $k^{-1} \mathbf{u}^{n},(1.3)$ by $\mu$, and add the resulting equations. The convective term in (1.1) disappears (cf. [23, Ch. 5, Lemma 2.9]). The convective term in (1.3) cancels out with the coupling term on the right-hand side of (1.1). Using (2.3) and Korn's inequality, we arrive at

$$
\frac{\mathrm{d}}{\mathrm{d} t} E\left[\mathbf{u}^{n}, \phi^{n}\right]+c_{1} v_{*}\left\|\nabla \mathbf{u}^{n}\right\|_{2}^{2}+c_{2} v_{*}\left\|\nabla \mathbf{u}^{n}\right\|_{p}^{p}+c_{3}\|\nabla \mu\|_{2}^{2} \leqslant c_{4}\|\mathbf{g}\|_{\left(W_{d i v}^{1,2}\right)^{*}}^{2},
$$

where

$$
E[\mathbf{u}, \phi]:=\frac{1}{k}\|\mathbf{u}\|_{2}^{2}+\varepsilon\|\nabla \phi\|_{2}^{2}+2 \alpha \int_{\Omega} F(\phi) \mathrm{d} x .
$$

We readily deduce that

$$
\left\{\mathbf{u}^{n}\right\} \text { is bounded in } L^{\infty}\left(0, T ; L_{\text {div }}^{2}\right) \cap L^{p}\left(0, T ; W_{\text {div }}^{1, p}\right) \text {. }
$$

By Poincaré's inequality, since $\overline{\phi^{n}} \equiv \overline{\phi^{n}}(0)$ is bounded, we further see that

$$
\left\{\phi^{n}\right\} \text { is bounded in } L^{\infty}\left(0, T ; H_{p e r}^{1}\right) .
$$

Similarly, if $N=3$, we have

$$
\begin{aligned}
\left\|\mu^{n}\right\|_{H_{p e r}^{1}} & \leqslant c\left(\left\|\nabla \mu^{n}\right\|_{2}^{2}+\left|\overline{F^{\prime}\left(\phi^{n}\right)}\right|\right) \\
\mid \overline{F^{\prime}\left(\phi^{n}\right) \mid} & \leqslant C_{F} \int_{\Omega}\left(1+\left|\phi^{n}\right|^{4}\right) \mathrm{d} x \leqslant C_{F}\left(1+\left\|\phi^{n}\right\|_{H_{p e r}^{1}}^{4}\right) .
\end{aligned}
$$

Hence we find that

$$
\left\{\mu^{n}\right\} \text { is bounded in } L^{2}\left(0, T ; H_{p e r}^{1}\right) \text {. }
$$


On the other hand, we have

$$
\begin{aligned}
c^{-1}\left\|\phi^{n}\right\|_{H_{p e r}^{3}} & \leqslant\left\|\nabla \mu^{n}\right\|_{2}+\left\|F^{\prime \prime}\left(\phi^{n}\right) \nabla \phi^{n}\right\|_{2}+\left|\overline{\phi^{n}}\right|, \\
\left\|F^{\prime \prime}\left(\phi^{n}\right) \nabla \phi^{n}\right\|_{2} & \leqslant\left\|F^{\prime \prime}\left(\phi^{n}\right)\right\|_{2}\left\|\nabla \phi^{n}\right\|_{\infty} \leqslant C_{F}\left(1+\left\|\phi^{n}\right\|_{6}^{3}\right)\left\|\phi^{n}\right\|_{H_{p e r}^{1}}^{1 / 4}\left\|\phi^{n}\right\|_{H_{p e r}^{3}}^{3 / 4} .
\end{aligned}
$$

Here (and below), we frequently use the interpolation inequalities

$$
\|\varphi\|_{\infty} \leqslant c\|\varphi\|_{H_{p e r}^{1}}^{3 / 4}\|\varphi\|_{H_{p e r}^{3}}^{1 / 4}, \quad\|\nabla \varphi\|_{\infty} \leqslant c\|\varphi\|_{H_{p e r}^{1}}^{1 / 4}\|\varphi\|_{H_{p e r}^{3}}^{3 / 4}
$$

as well as the embedding $H_{p e r}^{1} \hookrightarrow L^{6}$. We eventually find that

$$
\left\{\phi^{n}\right\} \text { is bounded in } L^{2}\left(0, T ; H_{p e r}^{3}\right) .
$$

With this information at hand, we come to a subsequence (not relabelled) such that

$$
\begin{array}{cl}
\mathbf{u}^{n} \rightarrow \mathbf{u} & \text { weakly in } L^{p}\left(0, T ; W_{d i v}^{1, p}\right), \\
\phi^{n} \rightarrow \phi & \text { weakly in } L^{2}\left(0, T ; H_{p e r}^{3}\right), \\
\mu^{n} \rightarrow \mu & \text { weakly in } L^{2}\left(0, T ; H_{p e r}^{1}\right), \\
\partial_{t} \mathbf{u}^{n} \rightarrow \partial_{t} \mathbf{u} & \text { weakly in } L^{p^{\prime}}\left(0, T ;\left(W_{d i v}^{1, p}\right)^{*}\right), \\
\partial_{t} \phi^{n} \rightarrow \partial_{t} \phi & \text { weakly in } L^{2}\left(0, T ;\left(H_{p e r}^{1}\right)^{*}\right) .
\end{array}
$$

In virtue of the Aubin-Lions lemma, we also have

$$
\begin{array}{ll}
\mathbf{u}^{n} \rightarrow \mathbf{u} & \text { strongly in } L^{p}\left(0, T ; L^{s}\right) \forall s \in[1,3 p /(3-p)), \\
\mathbf{u}^{n} \rightarrow \mathbf{u} & \text { in } C\left([0, T] ;\left(L_{\text {div }}^{2}\right)_{\mathrm{weak}}\right), \\
\phi^{n} \rightarrow \phi & \text { strongly in } L^{2}\left(0, T ; H_{\text {per }}^{3-\epsilon}\right) \quad \forall \epsilon>0 .
\end{array}
$$

Here the first convergence is for all $s \geqslant 1$ if $p \geqslant 3$. It is standard to obtain the limit in all the lower order nonlinearities; thus we will only treat the stress tensor. From (2.3) and (2.13) it follows that

$$
\left\langle N\left(\phi^{n}, \mathbf{e}(\mathbf{v})\right)-N\left(\phi^{n}, \mathbf{e}\left(\mathbf{u}^{n}\right)\right), \mathbf{v}-\mathbf{u}^{n}\right\rangle \geqslant 0 .
$$

Then we obtain (cf. (2.12))

$$
\left\langle\partial_{t} \mathbf{u}^{n}+N\left(\phi^{n}, \mathbf{e}(\mathbf{v})\right)+\left(\mathbf{u}^{n} \cdot \nabla\right) \mathbf{u}^{n}-k \mu^{n} \nabla \phi^{n}-\mathbf{g}, \mathbf{v}-\mathbf{u}^{n}\right\rangle \geqslant 0
$$

for an arbitrary smooth function $\mathbf{v}$. Thus we deduce

$$
\begin{aligned}
\left\langle\partial_{t} \mathbf{u}^{n}+N\left(\phi^{n}, \mathbf{e}(\mathbf{v})\right)+\right. & \left.\left(\mathbf{u}^{n} \cdot \nabla\right) \mathbf{u}^{n}-k \mu^{n} \nabla \phi^{n}-\mathbf{g}, \mathbf{v}\right\rangle \\
& \geqslant \frac{1}{2}\left\|u^{n}(T)\right\|_{2}^{2}-\frac{1}{2}\left\|u^{n}(0)\right\|_{2}^{2}+\left\langle N\left(\phi^{n}, \mathbf{e}(\mathbf{v})\right)-k \mu^{n} \nabla \phi^{n}-\mathbf{g}, \mathbf{u}^{n}\right\rangle .
\end{aligned}
$$

Taking the lower limit, we have

$$
\begin{aligned}
\left\langle\partial_{t} \mathbf{u}+N(\phi, \mathbf{e}(\mathbf{v}))+(\mathbf{u} \cdot \nabla) \mathbf{u}-\right. & k \mu \nabla \phi-\mathbf{g}, \mathbf{v}\rangle \\
& \geqslant \frac{1}{2}\|u(T)\|_{2}^{2}-\frac{1}{2}\|u(0)\|_{2}^{2}+\langle N(\phi, \mathbf{e}(\mathbf{v}))-k \mu \nabla \phi-\mathbf{g}, \mathbf{u}\rangle
\end{aligned}
$$


or

$$
\left\langle\partial_{t} \mathbf{u}+N(\phi, \mathbf{e}(\mathbf{v}))+(\mathbf{u} \cdot \nabla) \mathbf{u}-k \mu \nabla \phi-\mathbf{g}, \mathbf{v}-\mathbf{u}\right\rangle \geqslant 0 .
$$

At this stage, $\mathbf{v}$ can be replaced by an arbitrary function in $L^{p}\left(0, T ; W_{d i v}^{1, p}\right)$; using a standard argument (see, e.g., [27, Lemma 9.43]), we deduce that $\mathbf{u}$ is also solution to (1.1).

REMARK 2.5 Using a suitable approximating scheme, the above result readily gives the global existence of a weak solution (see also [18, Thm. 1]). For such a result, assumptions (2.1) can be weakened by taking, e.g., $F \in C^{2}(\mathbb{R} ; \mathbb{R})$.

It is well known that weak solutions-that is, having only regularity (2.6) - of the Ladyzhenskaya model are unique only if $p \geqslant(N+2) / 2$ (see [18, Thm. 3]), which is stronger than (2.5) for $N=3$. Thus, if we want to get uniqueness under (2.5), we have to improve the regularity of solutions (see $[18$, Sec. 6] for $N=2$ ).

THEOREM 2.6 Let $\mathbf{g} \in L_{d i v}^{2}$ and $\left(\mathbf{u}_{0}, \phi_{0}\right) \in W_{d i v}^{1,2} \times H_{p e r}^{2}$. Then there exists a weak solution with regularity

$$
\begin{aligned}
& \mathbf{u} \in L^{\infty}\left(0, T ; W_{d i v}^{1,2}\right) \cap L^{p}\left(0, T ; W_{d i v}^{1,3 p}\right) \cap L^{2}\left(0, T ; W_{d i v}^{2,2}\right), \\
& \phi \in L^{\infty}\left(0, T ; H_{p e r}^{2}\right) \cap L^{2}\left(0, T ; H_{p e r}^{4}\right) .
\end{aligned}
$$

Proof. Let us assume $N=3$. We again give a formal proof, which can be made rigorous at the level of suitable approximation. Rewrite (1.3)-(1.4) as

$$
\phi_{t}+\varepsilon \Delta^{2} \phi=-\mathbf{u} \cdot \nabla \phi+\alpha F^{(3)}(\phi)|\nabla \phi|^{2}+\alpha F^{\prime \prime}(\phi) \Delta \phi=: R_{1}+R_{2}+R_{3} .
$$

Testing with $\Delta^{2} \phi$ gives

$$
\frac{\mathrm{d}}{\mathrm{d} t}\|\Delta \phi\|_{2}^{2}+\varepsilon\left\|\Delta^{2} \phi\right\|_{2}^{2} \leqslant c \varepsilon^{-1} \int_{\Omega}\left(R_{1}^{2}+R_{2}^{2}+R_{3}^{2}\right) \mathrm{d} x .
$$

We begin with the estimate

$$
\int_{\Omega} R_{1}^{2} \mathrm{~d} x=\int_{\Omega}|\mathbf{u}|^{2}|\nabla \phi|^{2} \mathrm{~d} x \leqslant\|\mathbf{u}\|_{3}^{2}\|\nabla \phi\|_{6}^{2} \leqslant h_{1}(t)\|\phi\|_{H_{p e r}^{2}}^{2},
$$

where

$$
h_{1}(t):=c\|\nabla \mathbf{u}\|_{p}^{2}
$$

Furthermore, we have

$$
\int_{\Omega} R_{2}^{2} \mathrm{~d} x=\alpha^{2} \int_{\Omega}\left|F^{(3)}(\phi)\right|^{2}|\nabla \phi|^{4} \mathrm{~d} x \leqslant C_{F} \alpha^{2}\left(1+\|\phi\|_{12}^{4}\right)\|\nabla \phi\|_{6}^{4} .
$$

Using now the estimates

$$
\|\phi\|_{12} \leqslant c\|\phi\|_{H_{p e r}^{1}}^{7 / 8}\|\phi\|_{H_{p e r}^{3}}^{1 / 8}, \quad\|\nabla \phi\|_{6} \leqslant c\|\phi\|_{H_{p e r}^{2}} \leqslant \tilde{c}\|\phi\|_{H_{p e r}^{1}}^{1 / 2}\|\phi\|_{H_{p e r}^{3}}^{1 / 2},
$$

we obtain

$$
\int_{\Omega} R_{2}^{2} \mathrm{~d} x \leqslant h_{2}(t)\|\phi\|_{H_{p e r}^{2}}^{2}
$$


where

$$
h_{2}(t):=C_{F} \alpha^{2}\left(1+\|\phi\|_{H_{p e r}^{1}}^{9 / 2}\|\phi\|_{H_{p e r}^{3}}^{3 / 2}\right) .
$$

Finally, we observe that

$$
\int_{\Omega} R_{3}^{2} \mathrm{~d} x=\alpha^{2} \int_{\Omega}\left|F^{\prime \prime}(\phi)\right|^{2}|\Delta \phi|^{2} \mathrm{~d} x \leqslant C_{F} \alpha^{2}\left(1+\|\phi\|_{\infty}^{6}\right)\|\Delta \phi\|_{2}^{2} \leqslant h_{3}(t)\|\phi\|_{H_{p e r}^{2}}^{2},
$$

where

$$
h_{3}(t):=C_{F} \alpha^{2}\left(1+\|\phi\|_{H_{p e r}^{1}}^{9 / 2}\|\phi\|_{H_{p e r}^{3}}^{3 / 2}\right) .
$$

Recalling Poincaré's inequality and the fact that $\bar{\phi}$ is a constant of motion, we eventually have

$$
\frac{\mathrm{d}}{\mathrm{d} t}\|\phi\|_{H_{p e r}^{2}}^{2}+\varepsilon\|\phi\|_{H_{p e r}^{4}}^{2} \leqslant H_{1}(t)\|\phi\|_{H_{p e r}^{2}}^{2}
$$

where $H_{1}(t):=c \varepsilon\left(h_{1}(t)+h_{2}(t)+h_{3}(t)+1\right)$. Hence (2.20) follows by Gronwall's lemma.

Further, arguing formally, we test (1.1) with $-\Delta \mathbf{u}$. More precisely, we test $\partial^{2} \mathbf{u} / \partial x_{k}^{2}$ and sum the resulting equation over $k$. Obviously,

$$
\mathbf{e}\left(\frac{\partial}{\partial x_{k}} \mathbf{u}\right)=\frac{\partial}{\partial x_{k}} \mathbf{e}(\mathbf{u})
$$

Hence, from the stress tensor term, integrating by parts, we obtain

$$
\begin{aligned}
& I(\phi, \mathbf{e}(\mathbf{u})):=\int_{\Omega} \tau(\phi, \mathbf{e}(\mathbf{u})): \mathbf{e}(-\Delta \mathbf{u}) \mathrm{d} x=-\int_{\Omega} \tau(\phi, \mathbf{e}(\mathbf{u})): \frac{\partial}{\partial x_{k}} \mathbf{e}\left(\frac{\partial}{\partial x_{k}} \mathbf{u}\right) \mathrm{d} x \\
& =\int_{\Omega} \partial_{\mathbf{e}(\mathbf{u})} \boldsymbol{\tau}(\phi, \mathbf{e}(\mathbf{u})):\left(\mathbf{e}\left(\frac{\partial}{\partial x_{k}} \mathbf{u}\right) \otimes \mathbf{e}\left(\frac{\partial}{\partial x_{k}} \mathbf{u}\right)\right) \mathrm{d} x+\int_{\Omega}\left(\partial_{\phi} \boldsymbol{\tau}(\phi, \mathbf{e}(\mathbf{u})) \frac{\partial}{\partial x_{k}} \phi\right): \mathbf{e}\left(\frac{\partial}{\partial x_{k}} \mathbf{u}\right) \mathrm{d} x
\end{aligned}
$$

In the former integral, with a slight abuse of notation, : stands for the product of two fourth-order tensors. Writing everything in terms of components, one sees that

$$
\partial_{\mathbf{e}(\mathbf{u})} \tau(\phi, \mathbf{e}(\mathbf{u})):(\mathbb{A} \otimes \mathbb{A})=\partial_{\mathbf{e}(\mathbf{u})_{i j}} \tau(\phi, \mathbf{e}(\mathbf{u}))_{m n} \mathbb{A}_{i j} \mathbb{A}_{m n} \geqslant c\left(v_{1}\left(\phi_{1}\right)+v_{2}(\phi)|\mathbf{e}(\mathbf{u})|^{p-2}\right)|\mathbb{A}|^{2}
$$

for any symmetric tensor $\mathbb{A} \in \mathbb{R}^{3 \times 3}$. Thus we have

$$
I(\phi, \mathbf{e}(\mathbf{u})) \geqslant \int_{\Omega} \mathbf{J}_{p}(\phi, \mathbf{u}) \mathrm{d} x-c v^{\sharp} \int_{\Omega}(1+|\mathbf{e}(\mathbf{u})|)^{p-1}\left|\mathbf{e}\left(\frac{\partial}{\partial x_{k}} \mathbf{u}\right)\right||\nabla \phi| \mathrm{d} x,
$$

where

$$
\mathbf{J}_{p}(\phi, \mathbf{u}):=\left(v_{1}\left(\phi_{1}\right)+v_{2}(\phi)|\mathbf{e}(\mathbf{u})|^{p-2}\right)\left|\mathbf{e}\left(\frac{\partial}{\partial x_{k}} \mathbf{u}\right)\right|^{2}
$$

with the implicit summation over $k$. We recall that the following estimate holds (see [23, Ch. 5, Lemma 3.24]):

$$
\int_{\Omega} \mathbf{J}_{p}(\phi, \mathbf{u}) \mathrm{d} x \geqslant c v_{*} \int_{\Omega}\left(1+|\mathbf{e}(\mathbf{u})|^{p-2}\right)\left|\mathbf{e}\left(\frac{\partial}{\partial x_{k}} \mathbf{u}\right)\right|^{2} \mathrm{~d} x \geqslant c_{1} v_{*}\left(\|\mathbf{u}\|_{W^{2,2}}^{2}+\|\nabla \mathbf{u}\|_{3 p}^{p}\right) .
$$


On the other hand, using the well-known orthogonality of the convective term,

$$
\int_{\Omega} \frac{\partial}{\partial x_{k}}[(\mathbf{u} \cdot \nabla) \mathbf{u}]: \frac{\partial}{\partial x_{k}} \mathbf{u} \mathrm{d} x=\int_{\Omega}\left(\frac{\partial}{\partial x_{k}} \mathbf{u} \cdot \nabla\right) \mathbf{u}: \frac{\partial}{\partial x_{k}} \mathbf{u} \mathrm{d} x \leqslant\|\nabla \mathbf{u}\|_{3}^{3},
$$

while the right-hand side is estimated simply as

$$
\left|\int_{\Omega} \mathbf{g} \cdot(-\Delta \mathbf{u}) \mathrm{d} x\right| \leqslant \frac{c_{1}}{2} v_{*}\|\mathbf{u}\|_{W^{2,2}}^{2}+c v_{*}^{-1}\|\mathbf{g}\|_{2}^{2} .
$$

Altogether, we have deduced the following inequality:

$$
\begin{aligned}
\frac{\mathrm{d}}{\mathrm{d} t}\|\nabla \mathbf{u}\|_{2}^{2}+ & \mathbf{J}_{p}(\mathbf{u})+c_{1} v_{*}\|\mathbf{u}\|_{W^{2,2}}^{2}+c_{1} v_{*}\|\nabla \mathbf{u}\|_{3 p}^{p} \\
& \leqslant c_{2} v^{\sharp} \int_{\Omega}(1+|\mathbf{e}(\mathbf{u})|)^{p-1}|\mathbf{e}(\nabla \mathbf{u})||\nabla \phi| \mathrm{d} x+\|\nabla \mathbf{u}\|_{3}^{3}+\int_{\Omega}|\mu||\phi||\Delta \mathbf{u}| \mathrm{d} x+c v_{*}^{-1}\|\mathbf{g}\|_{2}^{2} \\
& =: P_{1}+P_{2}+P_{3}+c v_{*}^{-1}\|\mathbf{g}\|_{2}^{2} .
\end{aligned}
$$

Observe that

$$
P_{1} \leqslant \frac{1}{2} \int_{\Omega} \mathbf{J}_{p}(\mathbf{u}) \mathrm{d} x+c v_{*}^{-1} \nu^{\sharp 2} \int_{\Omega}(1+|\mathbf{e}(\mathbf{u})|)^{p}|\nabla \phi|^{2} \mathrm{~d} x,
$$

where the last integral is estimated by

$$
\|\nabla \phi\|_{6}^{2}\left(1+\|\nabla \mathbf{u}\|_{3 p / 2}^{p}\right) \leqslant c\|\phi\|_{H_{p e r}^{2}}^{2}\left(1+\|\nabla \mathbf{u}\|_{p}^{p / 2}\|\nabla \mathbf{u}\|_{3 p}^{p / 2}\right) \leqslant \frac{c_{1}}{2} \nu_{*}\|\nabla \mathbf{u}\|_{3 p}^{p}+h_{4}(t),
$$

with

$$
h_{4}(t):=c v_{*}^{-2} v^{\sharp 4}\|\phi\|_{H_{p e r}^{2}}^{4}\left(1+\|\nabla \mathbf{u}\|_{p}^{p}\right) .
$$

Similarly, we have

$$
P_{3} \leqslant \frac{c_{1}}{2} v_{*}\|\mathbf{u}\|_{W^{2,2}}^{2}+c v_{*}^{-1} \int_{\Omega}|\mu|^{2}|\nabla \phi|^{2} \mathrm{~d} x,
$$

where the integral is estimated by

$$
c v_{*}^{-1}\|\mu\|_{4}^{2}\|\nabla \phi\|_{4}^{2} \leqslant c v_{*}^{-1}\|\mu\|_{H_{p e r}^{1}}^{2}\|\phi\|_{H_{p e r}^{2}}^{2}=: h_{5}(t) .
$$

The most difficult term to handle is $P_{2}$, coming from the convective term. Here we slightly modify the technique of [23, Chap. 5, proof of Thm.3.4].

Let us assume $p<3$ first. Using the interpolation inequalities

$$
\begin{array}{ll}
\|v\|_{3} \leqslant\|v\|_{p}^{\alpha}\|v\|_{3 p}^{1-\alpha}, & \alpha=\frac{p-1}{2}, \\
\|v\|_{3} \leqslant\|v\|_{2}^{\beta}\|v\|_{3 p}^{1-\beta}, & \beta=\frac{2(p-1)}{3 p-2},
\end{array}
$$

we can write

$$
P_{2} \leqslant\|\nabla \mathbf{u}\|_{3}^{3(1-a)}\|\nabla \mathbf{u}\|_{3}^{3 a} \leqslant\|\nabla \mathbf{u}\|_{2}^{Q_{1}}\|\nabla \mathbf{u}\|_{p}^{Q_{2}}\|\nabla \mathbf{u}\|_{3 p}^{Q_{3}},
$$


where

$$
Q_{1}=\frac{6(1-a)(p-1)}{3 p-2}, \quad Q_{2}=\frac{3 a(p-1)}{2}, \quad Q_{3}=3\left[\frac{(1-a) p}{3 p-2}+\frac{a(3-p)}{2}\right] .
$$

We now apply Young's inequality with

$$
\frac{1}{\delta}+\frac{1}{\delta^{\prime}}=1, \quad \delta Q_{3}=p, \quad \delta^{\prime} Q_{1}=2 .
$$

These conditions determine the choice of $a$ and $\delta$, namely,

$$
a=\frac{4 p}{3(p-1)(5 p-6)}, \quad \delta=\frac{5 p-6}{3} .
$$

We finally deduce

$$
P_{2} \leqslant \frac{c_{1}}{4} v_{*}\|\nabla \mathbf{u}\|_{3 p}^{p}+H_{3}(t)\|\nabla \mathbf{u}\|_{2}^{2}
$$

where

$$
H_{3}(t):=c v_{*}^{-\frac{3}{5 p-9}}\|\nabla \mathbf{u}\|_{p}^{\frac{2 p}{5 p-9}}
$$

Observe that $2 p /(5 p-9) \leqslant p$ is equivalent to $p \geqslant 11 / 5$, and thus $H_{3}$ is integrable in time. Summing up, recalling (2.22), we come to

$$
\frac{\mathrm{d}}{\mathrm{d} t}\|\nabla \mathbf{u}\|_{2}^{2}+c_{2} v_{*}\|\mathbf{u}\|_{2,2}^{2}+c_{3} v_{*}\|\nabla \mathbf{u}\|_{3 p}^{p} \leqslant H_{2}(t)+H_{3}(t)\|\nabla \mathbf{u}\|_{2}^{2},
$$

where $H_{2}(t):=h_{4}(t)+h_{5}(t)$. Thus (2.19) follows by Gronwall's lemma.

The case $p \geqslant 3$ is simpler, since now $\|\nabla \mathbf{u}\|_{3}^{3}$ is integrable (see (2.14)). Hence (2.24) holds with $H_{2}=h_{4}+h_{5}+\|\nabla \mathbf{u}\|_{3}^{3}$ and $H_{3} \equiv 0$.

REMARK 2.7 In the case of simple fluids, following [8], if $N=3$ and

$$
p>12 / 5
$$

the regularity of solutions can be proven in the case $\mathbf{g} \in\left(W_{d i v}^{1,2}\right)^{*}$. This result is obtained by taking $\mathbf{u}_{t}$ in place of $-\Delta \mathbf{u}$ as a test function. It should be possible to extend [8, Thm. 3.3] to system (1.1)(1.4) provided that $v_{1}$ and $v_{2}$ are constants. If so, all the following results would hold under a more general (nongradient) external force. We recall that the longterm dynamics is strongly affected by the presence of such a force (see Remark 3.2 below). We also refer to [9] for an alternative approach to improving regularity of three-dimensional non-Newtonian fluids, based on estimates of fractional time differences, which works for $p>11 / 5$ and is independent of boundary conditions.

THEOREM 2.8 Any solution satisfying (2.19) and (2.20) is unique in the class of weak solutions. More precisely: given two solutions $\left(\mathbf{u}_{1}, \phi_{1}\right),\left(\mathbf{u}_{2}, \phi_{2}\right)$, we have

$$
\begin{aligned}
\frac{\mathrm{d}}{\mathrm{d} t}\left(\|\mathbf{w}\|_{2}^{2}+\|\psi\|_{H_{p e r}^{1}}^{2}\right)+\varepsilon\|\psi\|_{H_{p e r}^{3}}^{2}+c_{1} v_{*}\|\nabla \mathbf{w}\|_{2}^{2}+c_{1} v_{*} & \int_{\Omega} \mathbf{I}_{p}^{2}\left(\mathbf{e}\left(\mathbf{u}_{1}\right), \mathbf{e}\left(\mathbf{u}_{2}\right)\right) \mathrm{d} x \\
& \leqslant H_{4}(t)\left(\|\mathbf{w}\|_{2}^{2}+\|\psi\|_{H_{p e r}^{1}}^{2}\right),
\end{aligned}
$$


where $\mathbf{w}=\mathbf{u}_{1}-\mathbf{u}_{2}, \psi=\phi_{1}-\phi_{2}$, and

$$
\mathbf{I}_{p}\left(\mathbf{e}\left(\mathbf{u}_{1}\right), \mathbf{e}\left(\mathbf{u}_{2}\right)\right):=\left(1+\left|\mathbf{e}\left(\mathbf{u}_{1}\right)\right|+\left|\mathbf{e}\left(\mathbf{u}_{2}\right)\right|\right)^{p / 2-1}|\mathbf{e}(\mathbf{w})| .
$$

Here $H_{4} \in L^{1}(0, T)$ denotes a function only depending on the norms of $\left(\mathbf{u}_{2}, \phi_{2}\right)$ in the spaces specified in (2.6) and (2.7) and on the norms of ( $\left.\mathbf{u}_{1}, \phi_{1}\right)$ in the spaces specified in (2.19) and (2.20). Proof. We start with the equation for $\psi$, that is,

$$
\partial_{t} \psi+\left(\mathbf{u}_{1} \cdot \nabla \phi_{1}-\mathbf{u}_{2} \cdot \nabla \phi_{2}\right)+\varepsilon \Delta^{2} \psi-\alpha \Delta\left(F^{\prime}\left(\phi_{1}\right)-F^{\prime}\left(\phi_{2}\right)\right)=0 .
$$

Multiplying it by $-\Delta \psi$, which is an admissible test function, we arrive at

$$
\begin{aligned}
\frac{1}{2} \frac{\mathrm{d}}{\mathrm{d} t}\|\nabla \psi\|_{2}^{2}+\varepsilon\|\nabla \Delta \psi\|_{2}^{2} \leqslant & \int_{\Omega}\left(\mathbf{u}_{2} \phi_{2}-\mathbf{u}_{1} \phi_{1}\right) \cdot \nabla \Delta \psi \mathrm{d} x \\
& +\alpha \int_{\Omega} \nabla\left(F^{\prime}\left(\phi_{1}\right)-F^{\prime}\left(\phi_{2}\right)\right) \cdot \nabla \Delta \psi \mathrm{d} x=: I_{1}+I_{2} .
\end{aligned}
$$

The first term on the right-hand side is estimated as follows:

$$
I_{1} \leqslant \frac{\varepsilon}{8}\|\nabla \Delta \psi\|_{2}^{2}+c \varepsilon^{-1} \int_{\Omega}\left(|\mathbf{w}|^{2}\left|\phi_{1}\right|^{2}+\left|\mathbf{u}_{1}\right|^{2}|\psi|^{2}\right) \mathrm{d} x
$$

the integral being estimated by

$$
c \varepsilon^{-1}\left(\|\mathbf{w}\|_{2}^{2}\left\|\phi_{2}\right\|_{\infty}^{2}+\left\|\mathbf{u}_{1}\right\|_{3}^{2}\|\psi\|_{6}^{2}\right) \leqslant h_{6}(t)\left(\|\mathbf{w}\|_{2}^{2}+\|\psi\|_{H_{p e r}^{1}}^{2}\right),
$$

where

$$
h_{6}(t):=c \varepsilon^{-1}\left(\left\|\phi_{2}\right\|_{H_{p e r}^{1}}^{3 / 2}\left\|\phi_{2}\right\|_{H_{p e r}^{3}}^{1 / 2}+\left\|\nabla \mathbf{u}_{1}\right\|_{p}^{2}\right)
$$

Furthermore, we have

$$
I_{2} \leqslant \frac{\varepsilon}{8}\|\nabla \Delta \psi\|_{2}^{2}+c \varepsilon^{-1} \int_{\Omega}\left|\nabla\left(F^{\prime}\left(\phi_{1}\right)-F^{\prime}\left(\phi_{2}\right)\right)\right|^{2} \mathrm{~d} x .
$$

Observe now that we can write

$$
\begin{aligned}
F^{\prime}\left(\phi_{1}\right)-F^{\prime}\left(\phi_{2}\right)= & \int_{0}^{1} F^{\prime \prime}\left((1-s) \phi_{1}+s \phi_{2}\right) \mathrm{d} s \psi \\
\nabla\left(F^{\prime}\left(\phi_{1}\right)-F^{\prime}\left(\phi_{2}\right)\right)= & \int_{0}^{1} F^{(3)}\left((1-s) \phi_{1}+s \phi_{2}\right) \nabla\left((1-s) \phi_{1}+s \phi_{2}\right) \mathrm{d} s \psi \\
& +\int_{0}^{1} F^{\prime \prime}\left((1-s) \phi_{1}+s \phi_{2}\right) \mathrm{d} s \nabla \psi
\end{aligned}
$$

Consequently, recalling (2.1), we have

$$
\begin{aligned}
\int_{\Omega}\left|\nabla\left(F^{\prime}\left(\phi_{1}\right)-F^{\prime}\left(\phi_{2}\right)\right)\right|^{2} \mathrm{~d} x \leqslant & C_{F} \int_{\Omega}\left(1+\left|\phi_{1}\right|+\left|\phi_{2}\right|\right)^{4}\left(\left|\nabla \phi_{1}\right|+\left|\nabla \phi_{2}\right|\right)^{2}|\psi|^{2} \mathrm{~d} x \\
& +C_{F} \int_{\Omega}\left(1+\left|\phi_{1}\right|+\left|\phi_{2}\right|\right)^{6}|\nabla \psi|^{2} \mathrm{~d} x=: J_{1}+J_{2} .
\end{aligned}
$$


In view of (2.16), these integrals are then estimated as follows:

$$
\begin{aligned}
J_{1} & \leqslant C_{F}\left(1+\left\|\phi_{1}\right\|_{\infty}^{4}+\left\|\phi_{2}\right\|_{\infty}^{4}\right)\left(\left\|\nabla \phi_{1}\right\|_{2}^{2}+\left\|\nabla \phi_{2}\right\|_{2}^{2}\right)\|\psi\|_{\infty}^{2} \\
& \leqslant C_{F}\left(1+\left\|\phi_{1}\right\|_{H_{p e r}^{1}}^{5}+\left\|\phi_{2}\right\|_{H_{p e r}^{1}}^{5}\right)\left(\left\|\phi_{1}\right\|_{H_{p e r}^{3}}+\left\|\phi_{2}\right\|_{H_{p e r}^{3}}\right)\|\psi\|_{H_{p e r}^{1}}^{3 / 2}\|\psi\|_{H_{p e r}^{3}}^{1 / 2} \\
& \leqslant \frac{\varepsilon}{8}\|\psi\|_{H_{p e r}^{3}}^{2}+C_{F} \varepsilon^{-4 / 3}\left(1+\left\|\phi_{1}\right\|_{H_{p e r}^{1}}^{20 / 3}+\left\|\phi_{2}\right\|_{H_{p e r}^{1}}^{20 / 3}\right)\left(\left\|\phi_{2}\right\|_{H_{p e r}^{3}}^{4 / 3}+\left\|\phi_{2}\right\|_{H_{p e r}^{3}}^{4 / 3}\right)\|\psi\|_{H_{p e r}^{1}}^{2}, \\
J_{2} & \leqslant C_{F}\left(1+\left\|\phi_{1}\right\|_{6}^{6}+\left\|\phi_{2}\right\|_{6}^{6}\right)\|\nabla \psi\|_{\infty}^{2} \leqslant C_{F}\left(1+\left\|\phi_{1}\right\|_{H_{p e r}^{1}}^{6}+\left\|\phi_{2}\right\|_{H_{p e r}^{1}}^{6}\right)\|\psi\|_{H_{p e r}^{1}}^{1 / 2}\|\psi\|_{H_{p e r}^{3}}^{3 / 2} \\
& \leqslant \frac{\varepsilon}{8}\|\psi\|_{H_{p e r}^{3}}^{2}+C_{F} \varepsilon^{-4}\left(1+\left\|\phi_{1}\right\|_{H_{p e r}^{1}}^{24}+\left\|\phi_{2}\right\|_{H_{p e r}^{1}}^{24}\right)\|\psi\|_{H_{p e r}^{1}}^{2} .
\end{aligned}
$$

To summarize, we deduce that

$$
\frac{\mathrm{d}}{\mathrm{d} t}\|\psi\|_{H_{p e r}^{1}}^{2}+\varepsilon\|\psi\|_{H_{p e r}^{3}}^{2} \leqslant\left(h_{6}(t)+h_{7}(t)\right)\left(\|\mathbf{w}\|_{2}^{2}+\|\psi\|_{H_{p e r}^{1}}^{2}\right),
$$

where

$$
\begin{aligned}
h_{7}(t):= & C_{F} \varepsilon^{-4 / 3}\left(1+\left\|\phi_{1}\right\|_{H_{p e r}^{1}}^{20 / 3}+\left\|\phi_{2}\right\|_{H_{p e r}^{1}}^{20 / 3}\right)\left(\left\|\phi_{2}\right\|_{H_{p e r}^{3}}^{4 / 3}+\left\|\phi_{2}\right\|_{H_{p e r}^{3}}^{4 / 3}\right) \\
& +C_{F} \varepsilon^{-4}\left(1+\left\|\phi_{1}\right\|_{H_{p e r}^{1}}^{24}+\left\|\phi_{2}\right\|_{H_{p e r}^{1}}^{24}\right) .
\end{aligned}
$$

We proceed to handle the equation for $w$ which can be written as follows:

$$
\begin{aligned}
\partial_{t} \mathbf{w}+\mathbf{u}_{1} \cdot \nabla \mathbf{u}_{1}-\mathbf{u}_{2} \cdot \nabla \mathbf{u}_{2}-\nabla \cdot\left\{\boldsymbol{\tau}\left(\phi_{1}, \mathbf{e}\left(\mathbf{u}_{1}\right)\right)-\boldsymbol{\tau}\left(\phi_{2}, \mathbf{e}\left(\mathbf{u}_{2}\right)\right)\right\} & +\nabla\left(\pi_{1}-\pi_{2}\right) \\
& =k \mu_{1} \nabla \phi_{1}-k \mu_{2} \nabla \phi_{2} .
\end{aligned}
$$

Multiply it by $\mathbf{w}$ (an admissible test function due to (2.5)) to obtain

$$
\begin{aligned}
\frac{1}{2} \frac{\mathrm{d}}{\mathrm{d} t}\|\mathbf{w}\|_{2}^{2}+ & \underbrace{\int_{\Omega}\left\{\boldsymbol{\tau}\left(\phi_{2}, \mathbf{e}\left(\mathbf{u}_{1}\right)\right)-\tau\left(\phi_{2}, \mathbf{e}\left(\mathbf{u}_{2}\right)\right)\right\}: \mathbf{e}(\mathbf{w}) \mathrm{d} x}_{K_{1}} \\
= & \int_{\Omega}\left\{\boldsymbol{\tau}\left(\phi_{1}, \mathbf{e}\left(\mathbf{u}_{1}\right)\right)-\tau\left(\phi_{2}, \mathbf{e}\left(\mathbf{u}_{1}\right)\right)\right\}: \mathbf{e}(\mathbf{w}) \mathrm{d} x+\int_{\Omega}\left(\mathbf{u}_{2} \cdot \nabla \mathbf{u}_{2}-\mathbf{u}_{1} \cdot \nabla \mathbf{u}_{1}\right) \cdot \mathbf{w} \mathrm{d} x \\
& +k \int_{\Omega}\left(\mu_{2} \nabla \phi_{2}-\mu_{1} \nabla \phi_{1}\right) \cdot \mathbf{w} \mathrm{d} x=: K_{2}+K_{3}+K_{4}
\end{aligned}
$$

Thanks to (2.3) and Korn's inequality, we have

$$
K_{1} \geqslant c_{1} v_{*}\|\nabla \mathbf{w}\|_{2}^{2}+c_{1} v_{*} \int_{\Omega} \mathbf{I}_{p}^{2}\left(\mathbf{e}\left(\mathbf{u}_{1}\right), \mathbf{e}\left(\mathbf{u}_{2}\right)\right) \mathrm{d} x .
$$

On the other hand (cf. (2.3) once more)

$$
\begin{aligned}
K_{2} & \leqslant c_{2} v^{\sharp} \int_{\Omega}\left(1+\left|\mathbf{e}\left(\mathbf{u}_{1}\right)\right|\right)^{p-1}|\mathbf{e}(\mathbf{w})||\psi| \mathrm{d} x \leqslant \tilde{c}_{2} v^{\sharp} \int_{\Omega} \mathbf{I}_{p}\left(\mathbf{e}\left(\mathbf{u}_{1}\right), \mathbf{e}\left(\mathbf{u}_{2}\right)\right)\left(1+\left|\mathbf{e}\left(\mathbf{u}_{1}\right)\right|\right)^{p / 2}|\psi| \mathrm{d} x \\
& \leqslant \frac{c_{1} v_{*}}{4} \int_{\Omega} \mathbf{I}_{p}^{2}\left(\mathbf{e}\left(\mathbf{u}_{1}\right), \mathbf{e}\left(\mathbf{u}_{2}\right)\right) \mathrm{d} x+c_{3} v_{*}^{-1}\left(v^{\sharp}\right)^{2} \int_{\Omega}\left(1+\left|\nabla \mathbf{u}_{1}\right|^{p}\right)|\psi|^{2} \mathrm{~d} x .
\end{aligned}
$$


The second term is estimated as follows:

$$
c v_{*}^{-1}\left(v^{\sharp}\right)^{2}\left(1+\left\|\nabla \mathbf{u}_{1}\right\|_{3 p / 2}^{p}\right)\|\psi\|_{6}^{2} \leqslant h_{8}(t)\|\psi\|_{H_{p e r}^{1}}^{2},
$$

where

$$
h_{8}(t):=c v_{*}^{-1}\left(\nu^{\sharp}\right)^{2}\left(1+\left\|\nabla \mathbf{u}_{1}\right\|_{2}^{2 p /(3 p-2)}\left\|\nabla \mathbf{u}_{1}\right\|_{3 p}^{\left(3 p^{2}-4 p\right) /(3 p-2)}\right) .
$$

Here we have used the interpolation inequality

$$
\|v\|_{3 p / 2} \leqslant\|v\|_{2}^{2 /(3 p-2)}\|v\|_{3 p}^{(3 p-4) /(3 p-2)} .
$$

Furthermore, we insert $\pm \mathbf{u}_{2} \cdot \nabla \mathbf{u}_{1}$ and use again the orthogonality of convective terms to deduce

$$
K_{3}=-\int_{\Omega}\left(\mathbf{w} \cdot \nabla \mathbf{u}_{1}\right) \cdot \mathbf{w} \mathrm{d} x \leqslant\left\|\nabla \mathbf{u}_{1}\right\|_{3 p}\|\mathbf{w}\|_{6 p /(3 p-1)}^{2} .
$$

Here we have used the inequalities

$$
\|v\|_{6 p /(3 p-1)} \leqslant\|v\|_{2}^{a}\|v\|_{6}^{1-a} \leqslant c\|v\|_{2}^{a}\|\nabla v\|_{2}^{1-a}, \quad a=\frac{2 p-1}{2 p} .
$$

Therefore, we obtain

$$
K_{3} \leqslant c_{4}\left\|\nabla \mathbf{u}_{1}\right\|_{3 p}\|\mathbf{w}\|_{2}^{(2 p-1) / p}\|\nabla \mathbf{w}\|_{2}^{1 / p} \leqslant \frac{c_{1} v_{*}}{4}\|\nabla \mathbf{w}\|_{2}^{2}+h_{9}(t)\|\mathbf{w}\|_{2}^{2},
$$

where

$$
h_{9}(t):=c_{5} v_{*}^{-1 /(2 p-1)}\left\|\nabla \mathbf{u}_{1}\right\|_{3 p}^{2 p /(2 p-1)} .
$$

Note that the exponent $2 p /(2 p-1)$ is strictly smaller than $p$. We now use integration by parts and recall that $\mathbf{w}$ is divergence-free, to write

$$
K_{4}=k \int_{\Omega}\left(\nabla \mu_{1} \phi_{1}-\nabla \mu_{2} \phi_{2}\right) \cdot \mathbf{w} \mathrm{d} x=-k \int_{\Omega} \eta \nabla \phi_{2} \cdot \mathbf{w} \mathrm{d} x-k \int_{\Omega} \mu_{1} \nabla \psi \cdot \mathbf{w} \mathrm{d} x=: K_{4 a}+K_{4 b},
$$

where we have set $\eta:=\mu_{1}-\mu_{2}$. Then, we observe that

$$
\begin{aligned}
|\eta| & \leqslant \varepsilon|\Delta \psi|+\alpha C_{F}\left(1+\left|\phi_{1}\right|+\left|\phi_{2}\right|\right)^{3}|\psi|, \\
\|\eta\|_{2} & \leqslant \varepsilon\|\psi\|_{H_{p e r}^{2}}+\alpha C_{F}\left(1+\left\|\phi_{1}\right\|_{6}^{3}+\left\|\phi_{2}\right\|_{6}^{3}\right)\|\psi\|_{\infty} \\
& \leqslant c\left(\varepsilon+\alpha C_{F}\left(1+\left\|\phi_{1}\right\|_{H_{p e r}^{1}}^{3}+\left\|\phi_{2}\right\|_{H_{p e r}^{1}}^{3}\right)\|\psi\|_{H_{p e r}^{2}}\right.
\end{aligned}
$$

We thus have

$$
\begin{aligned}
K_{4 a} & \leqslant c k\left(\varepsilon+\alpha C_{F}\left(1+\left\|\phi_{1}\right\|_{H_{p e r}^{1}}^{3}+\left\|\phi_{1}\right\|_{H_{p e r}^{1}}^{3}\right)\|\psi\|_{H_{p e r}^{2}}\left\|\nabla \phi_{2}\right\|_{\infty}\|\mathbf{w}\|_{2}\right. \\
& \leqslant c k\left(\varepsilon+\alpha C_{F}\left(1+\left\|\phi_{1}\right\|_{H_{p e r}^{1}}^{3}+\left\|\phi_{1}\right\|_{H_{p e r}^{1}}^{3}\right)\|\psi\|_{H_{p e r}^{1}}^{1 / 2}\|\psi\|_{H_{p e r}^{3}}^{1 / 2}\left\|\phi_{2}\right\|_{H_{p e r}^{1}}^{1 / 4}\left\|\phi_{2}\right\|_{H_{p e r}^{3}}^{3 / 4}\|\mathbf{w}\|_{2}\right. \\
& \leqslant \frac{\varepsilon}{8}\|\psi\|_{H_{p e r}^{3}}^{2}+h_{10}(t)\left(\|\psi\|_{H_{p e r}^{1}}^{2}+\|\mathbf{w}\|_{2}^{2}\right),
\end{aligned}
$$


where

$$
h_{10}(t):=c k^{4 / 3} \varepsilon^{-1 / 3}\left(\varepsilon+\alpha C_{F}\left(1+\left\|\phi_{1}\right\|_{H_{p e r}^{1}}^{3}+\left\|\phi_{1}\right\|_{H_{p e r}^{1}}^{3}\right)\right)^{4 / 3}\left\|\phi_{2}\right\|_{H_{p e r}^{3}} .
$$

Finally, we note that

$$
|\mu| \leqslant \varepsilon|\Delta \phi|+\alpha C_{F}\left(1+|\phi|^{4}\right), \quad\|\mu\|_{2} \leqslant \varepsilon\|\phi\|_{H_{p e r}^{2}}+\alpha C_{F}\left(1+\|\phi\|_{8}^{4}\right) .
$$

Using the interpolation inequality

$$
\|\phi\|_{8} \leqslant c\|\phi\|_{H_{p e r}^{5 / 4}} \leqslant \tilde{c}\|\phi\|_{H_{p e r}^{1}}^{3 / 4}\|\phi\|_{H_{p e r}^{2}}^{1 / 4},
$$

we deduce

$$
\left\|\mu_{1}\right\|_{2} \leqslant\left(\varepsilon+\alpha C_{F}\left(1+\left\|\phi_{1}\right\|_{H_{p e r}^{1}}^{3}\right)\right)\left\|\phi_{1}\right\|_{H_{p e r}^{2}} .
$$

Hence, we find

$$
\begin{aligned}
K_{4 b} & \leqslant k\left\|\mu_{1}\right\|_{2}\|\nabla \psi\|_{\infty}\|\mathbf{w}\|_{2} \leqslant k\left\|\mu_{1}\right\|_{2}\|\psi\|_{H_{p e r}^{1}}^{1 / 4}\|\psi\|_{H_{p e r}^{3}}^{3 / 4}\|\mathbf{w}\|_{2} \\
& \leqslant \frac{\varepsilon}{8}\|\psi\|_{H_{p e r}^{3}}^{2}+h_{11}(t)\left(\|\mathbf{w}\|_{2}^{2}+\|\psi\|_{H_{p e r}^{1}}^{2}\right),
\end{aligned}
$$

where

$$
h_{11}(t):=c \varepsilon^{-3 / 5}\left(\varepsilon+\alpha C_{F}\left(1+\left\|\phi_{1}\right\|_{H_{p e r}^{1}}^{3}\right)\right)^{8 / 5}\left\|\phi_{1}\right\|_{H_{p e r}^{1}}^{4 / 5}\left\|\phi_{1}\right\|_{H_{p e r}^{3}}^{4 / 5} .
$$

Collecting the above estimates and invoking (2.29), we see that (2.26) holds with $H_{4}(t):=$ $\sum_{j=6}^{11} h_{j}(t)$, and this completes the proof.

A consequence of Theorem 2.8 is the following

COROLlaRY 2.9 Let $\left(\mathbf{u}_{i}, \phi_{i}\right), i=1,2$, be two weak solutions and suppose that $\left(\mathbf{u}_{1}, \phi_{1}\right)$ satisfies (2.19) and (2.20). Let $0<2 \ell \leqslant T$ be given. Then

$$
\begin{gathered}
\|\mathbf{w}(t)\|^{2}+\|\psi(t)\|_{H_{p e r}^{1}}^{2} \leqslant \lambda_{1}\left(\|\mathbf{w}(s)\|^{2}+\|\psi(s)\|_{H_{p e r}^{1}}^{2}\right), \quad 0 \leqslant s \leqslant t \leqslant T, \\
\|\mathbf{w}\|_{L^{2}\left(\ell, 2 \ell ; W_{d i v}^{1,2}\right)}^{2}+\|\psi\|_{L^{2}\left(\ell, 2 \ell ; H_{p e r}^{3}\right)}^{2}+\left\|\mathbf{I}_{p}\left(\mathbf{e}\left(\mathbf{u}_{1}\right), \mathbf{e}\left(\mathbf{u}_{2}\right)\right)\right\|_{L^{2}\left(\ell, 2 \ell ; L^{2}\right)} \\
\leqslant \lambda_{2}\left(\|\mathbf{w}\|_{L^{2}\left(0, \ell ; L_{d i v}^{2}\right)}^{2}+\|\psi\|_{L^{2}\left(0, \ell ; H_{p e r}^{1}\right)}^{2}\right),
\end{gathered}
$$

where $\lambda_{j}, j=1,2$, depend on $T$ and $\ell$, on the norms of $\left(\mathbf{u}_{2}, \phi_{2}\right)$ in the spaces specified in (2.6) and (2.7), on the norms of $\left(\mathbf{u}_{1}, \phi_{1}\right)$ in the spaces specified in (2.19) and (2.20) and on the other structural constants of the system.

Proof. For a fixed $s \in[0, T]$ we set

$$
\begin{aligned}
Y_{S}(t)= & \|\mathbf{w}(t)\|_{2}^{2}+\|\psi(t)\|_{H_{p e r}^{1}}^{2} \\
& +\int_{s}^{t}\left(\varepsilon\|\psi\|_{H_{p e r}^{3}}^{2}+c_{1} v_{*}\|\nabla \mathbf{w}\|_{2}^{2}\right)+c_{1} v_{*} \int_{s}^{t} \int_{\Omega} \mathbf{I}_{p}^{2}\left(\mathbf{e}\left(\mathbf{u}_{1}\right), \mathbf{e}\left(\mathbf{u}_{2}\right)\right) \mathrm{d} x
\end{aligned}
$$

for all $t \in[s, T]$. Now (2.26) can be written as $\frac{\mathrm{d}}{\mathrm{d} t} Y_{S}(t) \leqslant H_{4}(t) Y_{S}(t)$, which yields

$$
Y_{S}(t) \leqslant Y_{S}(s) \exp \left(\int_{s}^{t} H_{4}(\tau) \mathrm{d} \tau\right) .
$$


Thus (2.31) follows with $\lambda_{1}=\exp \left(\int_{0}^{T} H_{4}\right)$. In addition, if $s \in(0, \ell)$ and $t=2 \ell$, then we get

$$
\begin{aligned}
\int_{s}^{2 \ell}\left(\varepsilon\|\psi\|_{H_{p e r}^{3}}^{2}+c_{1} v_{*}\|\nabla \mathbf{w}\|_{2}^{2}\right)+c_{1} v_{*} \int_{s}^{2 \ell} & \int_{\Omega} \mathbf{I}_{p}^{2}\left(\mathbf{e}\left(\mathbf{u}_{1}\right), \mathbf{e}\left(\mathbf{u}_{2}\right)\right) \mathrm{d} x \\
& \leqslant\left(\|\mathbf{w}(s)\|_{2}^{2}+\|\psi(s)\|_{H_{p e r}^{1}}^{2}\right) \exp \left(\int_{s}^{2 \ell} H_{4}(\tau) \mathrm{d} \tau\right) .
\end{aligned}
$$

Integrating over $s \in(0, \ell)$ immediately gives (2.32) with $\lambda_{2}=\ell^{-1} \exp \left(\int_{0}^{2 \ell} H_{4}\right)$.

We also need the following Lipschitz estimate:

LEMMA 2.10 Let $0<\ell \leqslant T$ be fixed and let $\left(\mathbf{u}_{1}, \phi_{1}\right),\left(\mathbf{u}_{2}, \phi_{2}\right)$ be two weak solutions satisfying (2.19), (2.20). Set $\mathbf{w}=\mathbf{u}_{1}-\mathbf{u}_{2}$ and $\psi=\phi_{1}-\phi_{2}$. Then, for any $p \geqslant 11 / 5$, we have

$$
\begin{aligned}
& \left\|\partial_{t} \mathbf{w}\right\|_{L^{p^{\prime}}\left(0, \ell ;\left(W_{d i v}^{1, p}\right)^{*}\right)}+\left\|\partial_{t} \psi\right\|_{L^{2}\left(0, \ell ;\left(H_{p e r}^{1}\right)^{*}\right)} \\
& \quad \leqslant \Lambda\left\{\|\mathbf{w}\|_{L^{2}\left(0, \ell ; W_{d i v}^{1,2}\right)}+\left\|\mathbf{I}_{p}\left(\mathbf{e}\left(\mathbf{u}_{1}\right), \mathbf{e}\left(\mathbf{u}_{2}\right)\right)\right\|_{L^{2}\left(0, \ell ; L^{2}\right)}+\|\psi\|_{L^{2}\left(0, \ell ; H_{p e r}^{3}\right)}+\|\psi\|_{L^{\infty}\left(0, \ell ; H_{p e r}^{1}\right)}\right\},
\end{aligned}
$$

where $\Lambda$ is a computable constant, depending on the norms of the solutions in the spaces (2.19), (2.20).

Proof. We use the equation and a duality argument. First, recall that

$$
\left\|\partial_{t} \mathbf{w}\right\|_{L^{p^{\prime}}\left(0, \ell ;\left(W_{d i v}^{1, p}\right)^{*}\right)}=\sup _{\mathbf{z}}\left\{\int_{0}^{\ell}\left\langle\partial_{t} \mathbf{w}, \mathbf{z}\right\rangle \mathrm{d} t:\|\mathbf{z}\|_{L^{p}\left(0, \ell ; W_{d i v}^{1, p}\right)} \leqslant 1\right\} .
$$

Then, from (1.1) it follows that

$$
\begin{aligned}
\int_{0}^{\ell}\left\langle\partial_{t} \mathbf{w}, \mathbf{z}\right\rangle \mathrm{d} t= & \int_{\Omega \times(0, \ell)}\left(\left(\mathbf{u}_{2} \cdot \nabla\right) \mathbf{u}_{2}-\left(\mathbf{u}_{1} \cdot \nabla\right) \mathbf{u}_{1}\right) \cdot \mathbf{z} \mathrm{d} x \mathrm{~d} t \\
& +\int_{\Omega \times(0, \ell)}\left(\boldsymbol{\tau}\left(\phi_{2}, \mathbf{e}\left(\mathbf{u}_{2}\right)\right)-\tau\left(\phi_{1}, \mathbf{e}\left(\mathbf{u}_{1}\right)\right)\right): \nabla \mathbf{z} \mathrm{d} x \mathrm{~d} t \\
& +k \int_{\Omega \times(0, \ell)}\left(\mu_{1} \nabla \phi_{1}-\mu_{2} \nabla \phi_{2}\right) \cdot \mathbf{z} \mathrm{d} x \mathrm{~d} t=: P_{1}+P_{2}+P_{3} .
\end{aligned}
$$

Integrating by parts and using the fact that $\mathbf{u}$ is divergence-free, we have

$$
\begin{aligned}
P_{1} & =\int_{\Omega \times(0, \ell)}\left(\mathbf{u}_{1} \otimes \mathbf{u}_{1}-\mathbf{u}_{2} \otimes \mathbf{u}_{2}\right): \nabla \mathbf{z} \mathrm{d} x \mathrm{~d} t \leqslant \int_{\Omega \times(0, \ell)}\left(\left|\mathbf{u}_{1}\right|+\left|\mathbf{u}_{2}\right|\right)|\mathbf{w}||\nabla \mathbf{z}| \mathrm{d} x \mathrm{~d} t \\
& \leqslant \int_{0}^{\ell}\left(\left\|\mathbf{u}_{1}\right\|_{6 p /(5 p-6)}+\left\|\mathbf{u}_{2}\right\|_{6 p /(5 p-6)}\right)\|\mathbf{w}\|_{6}\|\nabla \mathbf{z}\|_{p} \mathrm{~d} t \leqslant L_{1}\left(\int_{0}^{\ell}\|\nabla \mathbf{w}\|_{2}^{2}\right)^{1 / 2},
\end{aligned}
$$

where

$$
L_{1}:=c \ell^{(p-2) /(2 p)} \sup _{t \in(0, \ell)}\left(\left\|\mathbf{u}_{1}(t)\right\|_{6 p /(5 p-6)}+\left\|\mathbf{u}_{2}(t)\right\|_{6 p /(5 p-6)}\right) .
$$


This is indeed finite as $6 p /(5 p-6) \leqslant 6$, in view of $(2.19)$. Here we have also used the estimate

$$
\left(\int_{0}^{\ell}\|\nabla \mathbf{z}\|_{p}^{2}\right)^{1 / 2} \leqslant c \ell^{(p-2) /(2 p)}
$$

Furthermore, invoking (2.3), we get

$$
\begin{aligned}
P_{2} \leqslant & c v^{*} \int_{\Omega \times(0, \ell)}\left(1+\left|\mathbf{e}\left(\mathbf{u}_{1}\right)\right|+\left|\mathbf{e}\left(\mathbf{u}_{2}\right)\right|\right)^{p-2}|\mathbf{e}(\mathbf{w})||\nabla \mathbf{z}| \mathrm{d} x \mathrm{~d} t \\
& +c v^{\sharp} \int_{\Omega \times(0, \ell)}\left(1+\left|\mathbf{e}\left(\mathbf{u}_{1}\right)\right|\right)^{p-1}|\psi||\nabla \mathbf{z}| \mathrm{d} x \mathrm{~d} t=: P_{2 a}+P_{2 b} .
\end{aligned}
$$

Observe now that

$$
\begin{aligned}
P_{2 a} & \leqslant c v^{*} \int_{\Omega \times(0, \ell)} \mathbf{I}_{p}\left(\mathbf{e}\left(\mathbf{u}_{1}\right), \mathbf{e}\left(\mathbf{u}_{2}\right)\right)\left(1+\left|\mathbf{e}\left(\mathbf{u}_{1}\right)\right|+\left|\mathbf{e}\left(\mathbf{u}_{2}\right)\right|\right)^{p / 2-1}|\nabla \mathbf{z}| \mathrm{d} x \mathrm{~d} t \\
& \leqslant c v^{*} \int_{0}^{\ell}\left\|\mathbf{I}_{p}\left(\mathbf{e}\left(\mathbf{u}_{1}\right), \mathbf{e}\left(\mathbf{u}_{2}\right)\right)\right\|_{2}\left(1+\left\|\nabla \mathbf{u}_{1}\right\|_{p}+\left\|\nabla \mathbf{u}_{2}\right\|_{p}\right)^{p / 2-1}\|\nabla \mathbf{z}\|_{p} \mathrm{~d} t \\
& \leqslant L_{2 a}\left(\int_{0}^{\ell}\left\|\mathbf{I}_{p}\left(\mathbf{e}\left(\mathbf{u}_{1}\right), \mathbf{e}\left(\mathbf{u}_{2}\right)\right)\right\|_{2}^{2}\right)^{1 / 2}
\end{aligned}
$$

where

$$
L_{2 a}=c v^{*}\left(\int_{0}^{\ell}\left(1+\left\|\nabla \mathbf{u}_{1}\right\|_{p}^{p}+\left\|\nabla \mathbf{u}_{2}\right\|_{p}^{p}\right) \mathrm{d} t\right)^{(p-2) / 2 p} .
$$

Also, setting $\rho:=6 p(p-1) /(5 p-6)$, we have

$$
P_{2 b} \leqslant c v^{\sharp} \int_{0}^{\ell}\left(1+\left\|\nabla \mathbf{u}_{1}\right\|_{\rho}\right)^{p-1}\|\psi\|_{6}\|\nabla \mathbf{z}\|_{p} \mathrm{~d} t \leqslant L_{2 b} \sup _{t \in(0, \ell)}\|\psi(t)\|_{H_{p e r}^{1}},
$$

where

$$
L_{2 b}=c v^{\sharp}\left(\int_{0}^{\ell}\left(1+\left\|\nabla \mathbf{u}_{1}(t)\right\|_{\rho}^{p}\right) \mathrm{d} t\right)^{(p-1) / p},
$$

and this quantity is finite, thanks to (2.19) and $\rho \leqslant 3 p$. The last term can be estimated as follows:

$$
P_{3} \leqslant k \int_{\Omega \times(0, \ell)}\left|\mu_{1}-\mu_{2}\right|\left|\nabla \phi_{1}\right||\mathbf{z}| \mathrm{d} x \mathrm{~d} t+k \int_{\Omega \times(0, \ell)}\left|\mu_{2}\right||\nabla \psi||\mathbf{z}| \mathrm{d} x \mathrm{~d} t:=P_{3 a}+P_{3 b}
$$

In this case, we obtain

$$
P_{3 a} \leqslant k \int_{0}^{\ell}\left\|\mu_{1}-\mu_{2}\right\|_{2}\left\|\nabla \phi_{1}\right\|_{3}\|\mathbf{z}\|_{6} \mathrm{~d} t \leqslant L_{3 a}\left(\int_{0}^{\ell}\|\psi\|_{H_{p e r}^{3}}^{2}\right)^{1 / 2},
$$

where, in view of (2.30) and (2.35), we have set

$$
L_{3 a}=c k \ell^{(p-2) /(2 p)} \sup _{t \in(0, \ell)}\left[\left(\varepsilon+\alpha C_{F}\left(\left\|\phi_{1}(t)\right\|_{H_{p e r}^{1}}^{3}+\left\|\phi_{2}(t)\right\|_{H_{p e r}^{1}}^{3}\right)\right)\left\|\nabla \phi_{1}(t)\right\|_{3}\right] .
$$


On the other hand, we get

$$
P_{3 b} \leqslant k \int_{0}^{\ell}\left\|\mu_{2}\right\|_{6 / 5}\|\nabla \psi\|_{\infty}\|\mathbf{z}\|_{6} \mathrm{~d} t \leqslant L_{3 b}\left(\int_{0}^{\ell}\|\psi\|_{H_{p e r}^{3}}^{2}\right)^{1 / 2},
$$

where

$$
L_{3 b}=c k \ell^{(p-2) /(2 p)} \sup _{t \in(0, \ell)}\left\|\mu_{2}(t)\right\|_{6 / 5} .
$$

Consider now the second term on the left-hand side of (2.34), that is,

$$
\left\|\partial_{t} \psi\right\|_{L^{2}\left(0, \ell ;\left(H_{p e r}^{1}\right)^{*}\right)}=\sup _{\omega}\left\{\int_{0}^{\ell}\left\langle\partial_{t} \psi, \omega\right\rangle \mathrm{d} t:\|\omega\|_{L^{2}\left(0, \ell ; H_{p e r}^{1}\right)} \leqslant 1\right\}
$$

and observe that, by (1.3),

$$
\begin{aligned}
\int_{0}^{\ell}\left\langle\partial_{t} \psi, \omega\right\rangle \mathrm{d} t= & \int_{\Omega \times(0, \ell)}\left(\mathbf{u}_{2} \phi_{2}-\mathbf{u}_{1} \phi_{1}\right) \cdot \nabla \omega \mathrm{d} x \mathrm{~d} t-\varepsilon \int_{\Omega \times(0, \ell)} \nabla(\Delta \psi) \cdot \nabla \omega \mathrm{d} x \mathrm{~d} t \\
& +\alpha \int_{\Omega \times(0, \ell)} \nabla\left(F^{\prime}\left(\phi_{1}\right)-F^{\prime}\left(\phi_{2}\right)\right) \cdot \nabla \omega \mathrm{d} x \mathrm{~d} t=: R_{1}+R_{2}+R_{3} .
\end{aligned}
$$

Here we have

$$
\begin{aligned}
R_{1} & \leqslant \int_{\Omega \times(0, \ell)}\left(|\mathbf{w}|\left|\phi_{1}\right|+\left|\mathbf{u}_{2}\right||\psi|\right)|\nabla \omega| \mathrm{d} x \mathrm{~d} t \leqslant \int_{0}^{\ell}\left(\|\mathbf{w}\|_{6}\left\|\phi_{1}\right\|_{3}+\left\|\mathbf{u}_{2}\right\|_{2}\|\psi\|_{\infty}\right)\|\nabla \omega\|_{2} \mathrm{~d} t \\
& \leqslant L_{4}\left\{\left(\int_{0}^{\ell}\|\mathbf{w}\|_{W_{d i v}^{1,2}}^{2} \mathrm{~d} t\right)^{1 / 2}+\left(\int_{0}^{\ell}\|\psi\|_{H_{\text {per }}^{3}}^{2} \mathrm{~d} t\right)^{1 / 2}\right\},
\end{aligned}
$$

where

$$
L_{4}=c \sup _{t \in(0, \ell)}\left(\left\|\phi_{1}(t)\right\|_{H_{p e r}^{1}}+\left\|\mathbf{u}_{2}(t)\right\|_{2}\right)
$$

Similarly, we find

$$
R_{2} \leqslant \varepsilon \int_{\Omega \times(0, \ell)}\left|\nabla^{3} \psi\right||\nabla \omega| \mathrm{d} x \mathrm{~d} t \leqslant c \varepsilon\left(\int_{0}^{\ell}\|\psi\|_{H_{p e r}^{3}}^{2} \mathrm{~d} t\right)^{1 / 2} .
$$

Also, recalling (2.28), we have

$$
\begin{aligned}
\int_{\Omega}\left|\nabla\left(F^{\prime}\left(\phi_{1}\right)-F^{\prime}\left(\phi_{2}\right)\right)\right|^{2} \mathrm{~d} x \leqslant & C_{F}\left(1+\left\|\phi_{1}\right\|_{\infty}^{4}+\left\|\phi_{2}\right\|_{\infty}^{4}\right)\left(\left\|\nabla \phi_{1}\right\|_{2}^{2}+\left\|\nabla \phi_{2}\right\|_{2}^{2}\right)\|\psi\|_{\infty}^{2} \\
& +C_{F}\left(1+\left\|\phi_{1}\right\|_{6}^{6}+\left\|\phi_{2}\right\|_{6}^{6}\right)\|\nabla \psi\|_{\infty}^{2},
\end{aligned}
$$

so that

where

$$
R_{3} \leqslant \int_{0}^{\ell}\left\|\nabla\left(F^{\prime}\left(\phi_{1}\right)-F^{\prime}\left(\phi_{2}\right)\right)\right\|_{2}\|\nabla \omega\|_{2} \mathrm{~d} t \leqslant L_{5}\left(\int_{0}^{\ell}\|\psi\|_{H_{p e r}^{3}}^{2} \mathrm{~d} t\right)^{1 / 2}
$$

$$
\begin{aligned}
L_{5}=\sup _{t \in(0, \ell)}\left\{C _ { F } ( 1 + \| \phi _ { 1 } ( t ) \| _ { H _ { p e r } ^ { 1 } } ^ { 2 } + \| \phi _ { 2 } ( t ) \| _ { H _ { p e r } ^ { 1 } } ^ { 2 } ) \left(\left\|\phi_{1}(t)\right\|_{H_{p e r}^{2}}\right.\right. & \left.+\left\|\phi_{2}(t)\right\|_{H_{p e r}^{2}}\right) \\
& \left.+C_{F}\left(1+\left\|\phi_{1}(t)\right\|_{H_{p e r}^{1}}^{3 / 2}+\left\|\phi_{2}(t)\right\|_{H_{p e r}^{1}}^{3 / 2}\right)\right\} .
\end{aligned}
$$


We have thus proven (2.34) with

$$
\Lambda:=L_{1}+L_{2 a}+L_{2 b}+L_{3 a}+L_{3 b}+L_{4}+c \varepsilon+L_{5} .
$$

\section{Longtime behavior}

In this section we investigate the longtime behavior of the dynamics of our system. First of all, we prove a dissipative estimate (see Theorem 3.1). We then employ the short trajectory approach (cf. [22, 24]) to establish the existence of global attractor (see Theorem 3.3). This will be a simple consequence of the compactness result proven in Theorem 2.4 above. We also show that the dynamics of trajectories has a smoothing property; consequently, there exists an exponential attractor. Finally, we discuss how an upper bound on its fractal dimension depends on the physical parameters of the system.

THEOREM 3.1 Let (2.1) and (2.2) hold. Then any weak solution (u, $\phi$ ) satisfies the dissipative estimate, for all $t \geqslant 0$,

$\frac{\mathrm{d}}{\mathrm{d} t} E[\mathbf{u}, \phi]+\tilde{c}_{1} \kappa E[\mathbf{u}, \phi]+\tilde{c}_{1}\left(\frac{v_{*}^{1}}{k}\|\nabla \mathbf{u}\|_{2}^{2}+\frac{v_{*}^{2}}{k}\|\nabla \mathbf{u}\|_{p}^{p}+\|\nabla \mu\|_{2}^{2}\right) \leqslant \frac{\tilde{c}_{2}}{k v_{*}^{1}}\|\mathbf{g}\|_{-1,2}^{2}+\alpha \varepsilon \tilde{c}_{3}$,

where

$$
\kappa:=\min \left\{v_{*}^{1}, \varepsilon\right\},
$$

and $\tilde{c}_{i}, i=1,2$, are positive constants independent of the data, while $\tilde{c}_{3}>0$ depends on $\bar{\phi}$.

Proof. Let (u, $\phi)$ be an arbitrary weak solution. Testing (1.1) with $k^{-1} \mathbf{u}$ and (1.3) with $\mu$ yields

$$
\frac{1}{2} \frac{\mathrm{d}}{\mathrm{d} t} E[\mathbf{u}, \phi]+k^{-1} \int_{\Omega} \tau(\phi, \mathbf{e}(\mathbf{u})): \mathbf{e}(\mathbf{u}) \mathrm{d} x+\|\nabla \mu\|_{2}^{2} \leqslant k^{-1}|\langle\mathbf{g}, \mathbf{u}\rangle|
$$

By (2.3) and Poincaré's inequality, we find

$$
\int_{\Omega} \boldsymbol{\tau}(\phi, \mathbf{e}(\mathbf{u})): \mathbf{e}(\mathbf{u}) \mathrm{d} x \geqslant c_{1}\left(v_{*}^{1}\|\nabla \mathbf{u}\|_{2}^{2}+v_{*}^{2}\|\nabla \mathbf{u}\|_{p}^{p}\right) .
$$

On the other hand,

$$
|\langle\mathbf{g}, \mathbf{u}\rangle| \leqslant\|\mathbf{g}\|_{-1,2}\|\nabla \mathbf{u}\|_{2} \leqslant \frac{c_{1} v_{*}^{1}}{2}\|\nabla \mathbf{u}\|_{2}^{2}+\frac{1}{2 c_{1} v_{*}^{1}}\|\mathbf{g}\|_{-1,2}^{2}
$$

Altogether we thus obtain

$$
\frac{\mathrm{d}}{\mathrm{d} t} E[\mathbf{u}, \phi]+c_{1}\left(\frac{v_{*}^{1}}{k}\|\nabla \mathbf{u}\|_{2}^{2}+\frac{v_{*}^{2}}{k}\|\nabla \mathbf{u}\|_{p}^{p}\right)+\|\nabla \mu\|_{2}^{2} \leqslant \frac{1}{c_{1} k v_{*}^{1}}\|\mathbf{g}\|_{-1,2}^{2} .
$$

Testing now (1.4) with $\phi-\bar{\phi}$ gives

$$
\langle\mu, \phi-\bar{\phi}\rangle=\varepsilon\|\nabla \phi\|_{2}^{2}+\alpha \int_{\Omega} F^{\prime}(\phi)(\phi-\bar{\phi}) \mathrm{d} x
$$


Using Poincaré's inequality we get

$$
\begin{aligned}
\langle\mu, \phi-\bar{\phi}\rangle & =\langle\mu-\bar{\mu}, \phi-\bar{\phi}\rangle \leqslant\|\mu-\bar{\mu}\|_{2}\|\phi-\bar{\phi}\|_{2} \\
& \leqslant c_{2}\|\nabla \mu\|_{2}\|\nabla \phi\|_{2} \leqslant \frac{\varepsilon}{2}\|\nabla \phi\|_{2}^{2}+\frac{c_{2}^{2}}{2 \varepsilon}\|\nabla \mu\|_{2}^{2} .
\end{aligned}
$$

Moreover, we deduce from (2.1) that

$$
c_{3} \int_{\Omega} F(\phi) \mathrm{d} x \leqslant \int_{\Omega} F^{\prime}(\phi)(\phi-\bar{\phi}) \mathrm{d} x+c_{4}
$$

for some $c_{3} \in(0,1)$ and for some $c_{4}>0$ depending on $\bar{\phi}$ (see (2.8)). Finally, combining (3.3)-(3.5) yields

$$
c_{5} \varepsilon\left(\varepsilon\|\nabla \phi\|_{2}^{2}+2 \alpha \int_{\Omega} F(\phi) \mathrm{d} x\right) \leqslant\|\nabla \mu\|_{2}^{2}+c_{6} \alpha \varepsilon
$$

for some $c_{5}>0$ and for some $c_{6}>0$ depending on $\bar{\phi}$. Then, substituting into (3.2), one deduces (3.1).

REMARK 3.2 Note that, in the case of simple fluids (more generally, for convex $F(\cdot)$ where $c_{4}$ can be taken 0 in (3.5)), when there is no (nongradient) external force (i.e., $\mathbf{g}=\mathbf{0}$ ), inequality (3.1) implies that $\mathbf{0}$ is globally asymptotically stable. This still holds when $\|\mathbf{g}\|_{2}$ is small enough (cf. [10, Ch. II, Prop. 5.3] for the 2D Navier-Stokes equations), where $\mathbf{0}$ is replaced by the unique solution to the corresponding stationary problem.

We now have all the ingredients to construct a dynamical system and prove that it has a global attractor and an exponential attractor. We will also observe that the attractor's dimension can be estimated in a completely explicit way. We will only sketch the outline of such a computation, being satisfied with the (nontrivial) observation that all the estimates grow at most polynomially with the data. We recall that the standard method based on the estimate of the Lyapunov exponents cannot be used in this case since the differentiability of the semigroup solution is not known (cf. [8]).

Recall that by data we mean the constants $k, \varepsilon$ and $\alpha$, occurring in (1.1)-(1.4); the viscosity bounds $\nu_{*}, v^{*}$ and $v^{\sharp}$ (see (2.2)-(2.4)); the norm of the external force $\|\mathbf{g}\|_{2}$ and a generic positive constant $C_{F}$ related to the growth of the potential $F$. We also recall that, throughout the paper, we have used $c$ and $c_{i}$ to denote absolute (i.e., independent of the data) positive constants which may change from line to line.

From now on we set $N=3$ and (rescaling the space variable) $\Omega=(0,1)^{3}$. Thus the embedding constants are also absolute. As is well known, the embedding constants typically only depend on the size and the shape of domain. They can blow up, however, close to critical exponents, as for example with $W^{1,3} \subset L^{q}, N=3$ and $q \rightarrow \infty$. We avoid such situations in our paper. We also point out that the following results can be reformulated for the case $N=2$ with less restrictions.

We will now follow an abstract scheme of the "method of trajectories", as presented in Section 2 of [24]. In particular, we need to verify the assumptions (A1-A10), which will imply the desired results. For the reader's convenience, the statement of (A1-A10) will be recalled, with the notation slightly adapted to the present setting. We will also invoke some general abstract results from [11, Ch. 2].

Let $\mathbf{g} \in L_{d i v}^{2}$ and set

$$
X:=L_{d i v}^{2} \times H_{\text {per }}^{1}, \quad X^{M}:=\left\{(\mathbf{v}, \psi) \in L_{d i v}^{2} \times H_{p e r}^{1}:|\bar{\psi}| \leqslant M\right\},
$$


for some given $M \geqslant 0$. This is a complete metric space with respect to the metric induced by the $X$-norm. Then, for any fixed $\tau>0$, we introduce the spaces

$$
\begin{aligned}
& X_{\tau}:=L^{2}(0, \tau ; X), \quad X_{\tau}^{M}:=L^{2}\left(0, \tau ; X^{M}\right), \\
& Y_{\tau}^{M}:=\left\{(\mathbf{v}, \psi) \in X_{\tau}^{M} \cap\left[L^{p}\left(0, \tau ; W_{d i v}^{1, p}\right)\right) \times L^{2}\left(0, \tau ; H_{p e r}^{3}\right)\right]:\left.\left(\mathbf{v}_{t}, \psi_{t}\right) \in L^{p^{\prime}}\left(0, \tau ;\left(W_{d i v}^{1, p}\right)^{*}\right) \times L^{2}\left(0, \tau ;\left(H_{p e r}^{1}\right)^{*}\right)\right\}, \\
& W_{\tau}^{M}:=\left\{(\mathbf{v}, \psi) \in X_{\tau}^{M} \cap\left[\left(L^{2}\left(0, \tau ; W_{d i v}^{1,2}\right)\right) \times L^{2}\left(0, \tau ; H_{p e r}^{3}\right)\right]:\right. \\
&\left.\quad\left(\mathbf{v}_{t}, \psi_{t}\right) \in L^{p^{\prime}}\left(0, \tau ;\left(W_{d i v}^{1, p}\right)^{*}\right) \times L^{2}\left(0, \tau ;\left(H_{p e r}^{1}\right)^{*}\right)\right\} .
\end{aligned}
$$

We clearly have $Y_{\tau}^{M} \hookrightarrow \hookrightarrow X_{\tau}^{M}$ and $W_{\tau}^{M} \hookrightarrow \hookrightarrow X_{\tau}^{M}$.

Recalling Theorem 2.4, Remark 2.5 and (2.11), we deduce that for an arbitrary initial condition in $X^{M}$ and arbitrary $T \in(0, \infty)$, there exists at least one solution belonging to $C\left([0, T] ; X_{\text {weak }}^{M}\right) \cap Y_{T}^{M}$. In other words, the assumption [24, (A1)] is satisfied. Moreover, by virtue of Theorem 3.1, the set

$$
B_{0}^{M}=\left\{(\mathbf{v}, \psi) \in X^{M}: E[\mathbf{v}, \psi] \leqslant R_{0}\right\}
$$

is uniformly absorbing and positively invariant, provided that $R_{0}>0$ is sufficiently large. This means that the assumption [24, (A2)] is satisfied as well. Note that $B_{0}^{M}$ is a closed set in $X^{M}$.

For any solution starting from $B_{0}^{M}$, arguing as in the proof of Theorem 2.4, one deduces

$$
\begin{gathered}
\sup _{t \in[0, T]}\left(\|\mathbf{u}(t)\|_{2}^{2}+\|\phi(t)\|_{H_{p e r}^{1}}^{2}\right)+\int_{0}^{T}\left(\|\mathbf{u}\|_{1, p}^{p}+\|\phi\|_{H_{p e r}^{3}}^{2}+\|\mu\|_{H_{p e r}^{1}}^{2}\right) \mathrm{d} t \\
\leqslant \Pi_{1}=\Pi_{1}(M)(T+1) .
\end{gathered}
$$

Here and in what follows, by $\Pi_{k}$ we denote an explicitly computable upper bound, depending polynomially on the data, values of $\Pi_{l}$ for $l<k$ and, possibly, on other quantities which will be pointed out.

We now fix $\ell>0$ and we consider the set $\mathcal{X}_{\ell}$ of $\ell$-trajectories, that is, of all weak solutions $(\mathbf{u}, \phi)$ given by Theorem 2.4 on the time interval $[0, \ell]$ such that $|\bar{\phi}(t)| \leqslant M$ for all $t \in[0, \ell]$. We endow $\mathcal{X}_{\ell}$ with the topology of $X_{\ell}$. Of course, each trajectory makes sense pointwise because of (2.11). Note, however, that $\mathcal{X}_{\ell}$ is not a complete metric space.

For the sake of studying large time dynamics, we shall focus on the set

$$
\mathcal{B}_{\ell}^{0}:=\left\{(\mathbf{u}, \phi) \in \mathcal{X}_{\ell}:(\mathbf{u}(0), \phi(0)) \in B_{0}^{M}\right\},
$$

consisting of all trajectories starting from $B_{0}^{M}$. Clearly, for each of such trajectories, there exists $\tau \in(0, \ell / 2)$ such that

$$
\|\mathbf{u}(\tau)\|_{1,2}^{2}+\|\phi(\tau)\|_{H_{p e r}^{2}}^{2} \leqslant 2 \Pi_{1}(M)\left(1+\ell^{-1}\right)=: \Pi_{2} .
$$

We are ready to apply Theorem 2.6. The key observation, however, is that the functions $H_{1}$ and $H_{3}$ (which only depend on the norms being controlled in (3.11)) are integrable with some power $\sigma>1$, depending on $p>11 / 5$ (cf. (2.23)). Hence, by taking $\ell$ small enough, namely $\ell \leqslant \Pi_{3}$, we ensure that

$$
\int_{0}^{2 \ell}\left(H_{1}+H_{3}\right) \mathrm{d} t \leqslant 1
$$


for any solution taking values in $B_{0}^{M}$. Therefore, when applying Gronwall's lemma to (2.21) and (2.24), the exponential terms are uniformly bounded by $\operatorname{exp(1).~Eliminating~thus~possible~}$ exponential dependence on the data, we obtain

$$
\sup _{t \in[\tau, \tau+2 \ell]}\left(\|\mathbf{u}(t)\|_{1,2}^{2}+\|\phi(t)\|_{H_{p e r}^{2}}^{2}\right)+\int_{\tau}^{\tau+2 \ell}\left(\|\mathbf{u}\|_{1,3 p}^{p}+\|\mathbf{u}\|_{2,2}^{2}+\|\phi\|_{H_{p e r}^{4}}^{2}\right) \mathrm{d} t \leqslant \Pi_{4} .
$$

By Theorem 2.8, this regularity also entails unique continuation of the trajectory after $t \geqslant \tau$, more precisely, for any $(\mathbf{v}, \psi) \in \mathcal{B}_{\ell}^{0}$ and any $T \geqslant \ell$, there exists only one solution on $[0, T]$ such that $\left.(\mathbf{u}, \phi)\right|_{[0, \ell]}=(\mathbf{v}, \psi)$. This just means that $[24,(\mathrm{~A} 3)]$ holds.

Consequently, one can introduce a semigroup $L_{t}: \mathcal{B}_{\ell}^{0} \rightarrow \mathcal{B}_{\ell}^{0}$ by setting

$$
\left\{L_{t}(\mathbf{v}, \psi)\right\}(s):=(\mathbf{u}(t+s), \phi(t+s)), \quad s \in[0, \ell] .
$$

We proceed to show that $L_{t}$ is Lipschitz continuous, which is the assumption [24, (A4)]. Indeed, if $(\mathbf{w}, \psi)$ is a difference of two solutions starting from $B_{0}^{M}$, then due to Corollary 2.9 and the above higher regularity on $[\tau, 2 \ell]$, we deduce that

$$
\|\mathbf{w}(s+\ell / 2)\|_{2}^{2}+\|\psi(s+\ell / 2)\|_{H_{p e r}^{1}}^{2} \leqslant \lambda_{1}\left(\|\mathbf{w}(s)\|_{2}^{2}+\|\psi(s)\|_{H_{p e r}^{1}}^{2}\right), \quad \forall s \in(\ell / 2, \ell) .
$$

Integrating over $s$ implies that $L_{t}$ is even uniformly Lipschitz continuous on $\mathcal{B}_{\ell}^{0}$ with respect to $t \in[0, T]$.

Furthermore, we claim that

$$
{\overline{\mathcal{B}_{\ell}^{0}}}^{X_{\ell}}=\mathcal{B}_{\ell}^{0}
$$

Since $\mathcal{B}_{\ell}^{0}$ is positively invariant with respect to the (continuous) operators $L_{t}$, (3.14) implies that ${\overline{L_{t} \mathcal{B}_{\ell}^{0}}}^{X} \subset \mathcal{B}_{\ell}^{0}$, and consequently [24, (A5)] is satisfied. Setting $\mathcal{B}_{\ell}^{1}={\overline{L_{\tau}\left(\mathcal{B}_{\ell}^{0}\right)}}^{X_{\ell}}$ for some $\tau \in$ $[\ell / 2, \ell]$ we obtain a compact invariant absorbing set.

Let us verify that (3.14) holds. Let $\left(\mathbf{v}_{n}, \psi_{n}\right) \in \mathcal{B}_{\ell}^{0}$, and let $\left(\mathbf{v}_{n}, \psi_{n}\right) \rightarrow(\mathbf{v}, \psi)$ strongly in $X_{\ell}$. Extracting a subsequence, we can assume that $\left(\mathbf{v}_{n}(t), \psi_{n}(t)\right) \rightarrow(\mathbf{v}(t), \psi(t))$ for almost any $t$, strongly in $X$. It follows by Theorem 2.4 that $(\mathbf{v}, \psi)$ is a weak solution, at least on $(\tau, \ell]$ for arbitrary $\tau>0$. Observe, furthermore, that $\left(\mathbf{v}_{n}, \psi_{n}\right)$ and hence $(\mathbf{v}, \psi)$ are bounded in the spaces (2.6-2.10), with $T=\ell$, and thus $(\mathbf{v}, \psi)$ is clearly a weak solution on $[0, \ell]$. Finally, from the continuity of $(\mathbf{v}, \psi)$ and the closedness and positive invariance of $B_{0}^{M}$, one concludes that $(\mathbf{v}(0), \psi(0)) \in B_{0}^{M}$.

Now, the assumptions (A1-A5) imply that the dynamical system of trajectories $\left(\mathcal{X}_{\ell}, L_{t}\right)$ has a global attractor $\mathcal{A}_{\ell}$ (see [24, Theorem 2.1]).

We will now deal with the attractor's fractal dimension. Due to Corollary 2.9 and Lemma 2.10, $L_{\tau}: \mathcal{X}_{\ell} \rightarrow W_{\ell}^{M}$ is Lipschitz continuous on $\mathcal{B}_{\ell}^{1}$, which is the assumption [24, (A6)]. Note that $W_{\ell}^{M}$ is compactly embedded in $X_{\ell}$; in other words, $L_{\tau}$ has the so-called smoothing property. Moreover, since $\ell$ and $\tau$ were chosen small enough, we control the Lipschitz constant of $L_{\tau}$, i.e.,

$$
\mathcal{L}:=\operatorname{Lip}\left(L_{\tau} \mid \mathcal{B}_{\ell}^{1} ; X_{\ell}^{M}, W_{\ell}^{M}\right) \leqslant \Pi_{5}
$$

It follows that the global attractor $\mathcal{A}_{\ell}$ has finite fractal dimension in $X_{\ell}^{M}$. See [24, Theorem 2.2]. More precisely, we have an explicit estimate (cf. [11, Theorem 2.18], for example)

$$
\operatorname{dim}_{f}^{X_{\ell}^{M}}\left(\mathcal{A}_{\ell}\right) \leqslant \log \mathcal{K} / \log 2,
$$


where

$$
\mathcal{K}:=N_{X_{\ell}^{M}}\left(B_{W_{\ell}^{M}}(0 ; 1), 1 / 4 \mathcal{L}\right)
$$

Here $\mathcal{K}$ denotes the minimal number of balls in $X_{\ell}^{M}$ that are needed to cover a unit ball in $W_{\ell}^{M}$. Finally, the number $\mathcal{K}$ can also be explicitly calculated (see [11, Appendix]) so that

$$
\log \mathcal{K} \leqslant \Pi_{6}(\mathcal{L})
$$

To bring the results back to the phase space $X^{M}$, we now consider the mapping $e: \mathcal{X}_{\ell} \rightarrow X^{M}$, $(\mathbf{u}, \phi) \mapsto(\mathbf{u}(\ell), \phi(\ell))$. Recalling Corollary 2.9 once more and arguing as in the proof of continuity of $L_{t}$ above (cf. also [24, Lemma 2.1(ii)]), we deduce that $e$ is Lipschitz continuous on $\mathcal{B}_{\ell}^{0}$. This tells us that the assumption [24, (A8)] is fulfilled. Therefore, invoking [24, Theorems 2.3, 2.4] we deduce that

$$
\mathcal{A}:=e\left(\mathcal{A}_{\ell}\right)
$$

is the global attractor of the dynamical system $\left(e\left(\mathcal{B}_{\ell}^{1}\right), S_{t}\right)$, where $S_{t}$ is the solution operator defined by $S_{t}\left(\mathbf{u}_{0}, \phi_{0}\right):=(\mathbf{u}(t), \phi(t))$ for all $t \geqslant 0$. Moreover, we have the estimate

$$
\operatorname{dim}_{f}^{X^{M}}(\mathcal{A}) \leqslant \operatorname{dim}_{f}^{X_{\ell}^{M}}\left(\mathcal{A}_{\ell}\right)
$$

Finally, we address the problem of an exponential attractor. The smoothing property, i.e., the Lipschitz continuity $L_{\tau}$ on $\mathcal{B}_{\ell}^{1}$ with respect to $X_{\ell}^{M}$ and $W_{\ell}^{M}$, implies the existence of an exponential attractor $\mathcal{E}_{\ell}^{*}$ for the discrete dynamical system $\left(\mathcal{B}_{\ell}^{1}, L_{n \tau}\right)$; moreover, the dimension of $\mathcal{E}_{\ell}^{*}$ admits the same upper bound as the global attractor $\mathcal{A}_{\ell}$. See [11, Proposition 2.26]. To extend the results to the full semigroup $L_{t}$, we need to estimate the modulus of continuity of $L_{t} \chi$ both with respect to $t$ and $\chi$.

We have already observed above that $L_{t}: \mathcal{B}_{\ell}^{1} \rightarrow \mathcal{B}_{\ell}^{1}$ is Lipschitz continuous, uniformly with respect to $t \in[0, \tau]$. Thus [24, (A9)] holds true. Furthermore, if $(\mathbf{u}, \phi)$ is the continuation of the trajectory from $\mathcal{B}_{\ell}^{1}$, it follows from the regularity of Theorem 2.6 and the equation that $\left(\partial_{t} \mathbf{u}, \partial_{t} \phi\right)$ is bounded in $L^{p^{\prime}}\left(0, \tau+\ell ;\left(W_{d i v}^{1, p}\right)^{*}\right) \times L^{2}\left(0, \tau+\ell ; L^{2}\right)$. This entails that $t \mapsto L_{t} \chi$ is Hölder continuous with the target space $L^{p^{\prime}}\left(0, \ell ;\left(W_{d i v}^{1, p}\right)^{*}\right) \times L^{2}\left(0, \ell ; L^{2}\right)$ (see, e.g., [24, Lemma 2.2]). Invoking finally the pointwise boundedness of trajectories in $W_{d i v}^{2,2} \times H_{p e r}^{2}$ and a suitable interpolation, we conclude that $t \mapsto L_{t} \chi$ is Hölder continuous into the desired target space $X_{\ell}^{M}$. Thus the assumption [24, (A10)] holds. This, in turn, gives exponential attractors for the dynamical systems $\left(\mathcal{X}_{\ell}, L_{t}\right)$ and eventually, $\left(e\left(\mathcal{B}_{\ell}^{1}\right), S_{t}\right)$; see [24, Theorems $\left.2.5,2.6\right]$.

These attractors admit the same (up to multiplying by an absolute constant) explicit estimate of fractal dimension as was the case for the global attractor. Summing up, we have proven the following

THEOREM 3.3 Let $\mathbf{g} \in L_{d i v}^{2}$. The dynamical system generated by (1.1)-(1.4) on $X^{M}$ has an exponential attractor and the global attractor of finite fractal dimension. Moreover, if $p>11 / 5$, then the dimension can be estimated by an explicitly computable constant which depends polynomially on the data.

REMARK 3.4 Recalling (2.23) and (3.13), we deduce that the length of the trajectory tends to 0 as $p$ approaches the critical value $p=11 / 5$. Therefore, in this case, we are not able to estimate the dimension in such a way that the dependence on the data is of polynomial type. 
REMARK 3.5 We recall that the global attractor $\mathcal{A}$ attracts the dynamics originating from the whole space $X^{M}$ in the following sense (see [24, Rem. 2.1]): for any bounded set $B \subset X^{M}$, indicating by $B_{t}$ the set of all values of all solutions to (1.1)-(1.4) emanating from $B$ at time $t$, we have $\operatorname{dist}_{X}\left(B_{t}, \mathcal{A}\right) \rightarrow 0$ as $t$ goes to $\infty$.

\section{Acknowledgements}

The authors thank the reviewers for their careful reading of the manuscript as well as for having pointed out a number of inaccuracies and obscure points.

This work originated from a visit of the first author to the Nečas Center for Mathematical Modeling (Prague, Czech Republic) whose support is gratefully acknowledged. The first author has also been partially supported by the the Italian MIUR-PRIN Research Project 2008 Transizioni di fase, isteresi e scale multiple. The second author has been supported by the project MSM 0021620839 of the Czech Ministry of Education and the project 201/08/0315 of GA ČR.

\section{REFERENCES}

1. Abels, H. On a diffuse interface model for two-phase flows of viscous, incompressible fluids with matched densities. Arch. Ration. Mech. Anal. 194 (2009), 463-506. Zbl pre05640833 MR 2563636

2. ABELS, H. Longtime behavior of solutions of a Navier-Stokes/Cahn-Hilliard system. In: Nonlocal and Abstract Parabolic Equations and their Applications (Będlewo, 2207), Banach Center Publ. 86, Inst. Math., Polish Acad. Sci. (2009), 9-19. Zbl 1167.76008 MR 2571478

3. Anderson, D. M., McFadden, G. B., \& Wheeler, A. A. Diffuse-interface methods in fluid mechanics. In: Annual Review of Fluid Mechanics 30, Annual Reviews, Palo Alto, CA (1998), 139-165. MR 1609626

4. Badalassi, V. E., Ceniceros, H. D., \& Banerjee, S. Computation of multiphase systems with phase field models. J. Comput. Phys. 190 (2003), 371-397. Zbl 1076.76517 MR 2013023

5. BEIRÃO DA VEIGA, H. On the Ladyzhenskaya-Smagorinsky turbulence model of the NavierStokes equations in smooth domains. The regularity problem. J. Eur. Math. Soc. 11 (2009), 127-167. Zbl 1155.35073 MR 2471134

6. BOYER, F. Mathematical study of multi-phase flow under shear through order parameter formulation. Asymptot. Anal. 20 (1999), 175-212. Zbl 0937.35123 MR 1700669

7. Boyer, F. Nonhomogeneous Cahn-Hilliard fluids. Ann. Inst. H. Poincaré Anal. Non Linéaire 18 (2001), 225-259. Zbl 1037.76062 MR 1808030

8. Bulíček, M., Ettwein, F., KAPlický, P., \& PražÁK, D. The dimension of the attractor for the 3D flow of a non-Newtonian fluid. Comm. Pure Appl. Anal. 8 (2009), 1503-1520. Zbl 1200.37074 MR 2505283

9. BulíčEK, M., EtTwein, F., KAPlickÝ, P., \& PraŽÁK, D. On uniqueness and time regularity of flows of power-law like non-Newtonian fluids. Math. Meth. Appl. Sci. 33 (2010), 1995-2010. Zbl 1202.35152 MR 2744616

10. ChepyZhov, V. V., \& VishiK, M. I. Attractors for Equations of Mathematical Physics. Amer. Math. Soc. Colloq. Publ. 49, Amer. Math. Soc., Providence, RI (2002). Zbl 0986.35001 MR 1868930

11. FeIreisl, E., \& PražÁK, D. Asymptotic Behavior of Dynamical Systems in Fluid Mechanics. Appl. Math. 4, AIMS, Springfield (2010). Zbl 1198.37002 MR 2657204

12. FENG, X. Fully discrete finite element approximation of the Navier-Stokes-Cahn-Hilliard diffuse interface model for two-phase flows. SIAM J. Numer. Anal. 44 (2006), 1049-1072. Zbl pre05167765 MR 2231855 
13. Gal, C. G., \& Grasselli, M. Asymptotic behavior of a Cahn-Hilliard-Navier-Stokes system in 2D. Ann. Inst. H. Poincaré Anal. Non Linéaire 27 (2010), 401-436. Zbl 1184.35055 MR 2580516

14. Gal, C. G., \& GRASselli, M. Trajectory attractors for binary fluid mixtures in 3D. Chinese Ann. Math. Ser. B 31 (2010), 655-678. Zbl 1223.35079 MR 2726061

15. Gurtin, M. E., Polignone, D., \& Viñals, J. Two-phase binary fluids and immiscible fluids described by an order parameter. Math. Models Methods Appl. Sci. 6 (1996), 815-831. Zbl 0857.76008 MR 1404829

16. Kay, D., Styles, V., \& Welford, R. Finite element approximation of a Cahn-Hilliard-NavierStokes system. Interfaces Free Bound. 10 (2008), 15-43. Zbl 1144.35043 MR 2383535

17. Kim, J., Kang, K., \& Lowengrub, J. Conservative multigrid methods for Cahn-Hilliard fluids. J. Comput. Phys. 193 (2004), 511-543. Zbl 1109.76348 MR 2030475

18. Kim, N., CONSiglieri, L., \& Rodrigues, J. F. On non-Newtonian incompressible fluids with phase transitions. Math. Methods Appl. Sci. 29 (2006), 1523-1541. Zbl 1101.76004 MR 2249576

19. Hohenberg, P. C., \& Halperin, B. I. Theory of dynamical critical phenomena. Rev. Modern Phys. 49 (1977), 435-479.

20. LADYZHENSKAYA, O. A. New equations for the description of the motions of viscous incompressible flows and global solvability for their boundary value problems. Trudy Mat. Inst. Steklov. 102 (1967), 85104 (in Russian). Zbl 0202.37802

21. LiU, C., \& ShEN, J. A phase field model for the mixture of two incompressible fluids and its approximation by a Fourier-spectral method. Phys. D 179 (2003), 211-228. Zbl 1092.76069 MR 1984386

22. MáleK, J., \& NeČAS, J. A finite-dimensional attractor for three-dimensional flow of incompressible fluids. J. Differential Equations 127 (1996), 498-518. Zbl 0851.35107 MR 1389407

23. MÁlek, J., NeČAs, J., Rokyta, M., \& RŮŽIČKA, M. Weak and Measure-Valued Solutions to Evolutionary PDEs. Appl. Math. Math. Comput. 13, Chapman \& Hall, London (1996). Zbl 0851.35002 MR 1409366

24. MÁlek, J., \& PraŽÁK, D. Large time behavior via the method of l-trajectories. J. Differential Equations 181 (2002), 243-279. Zbl 1187.37113 MR 1907143

25. MÁleK, J., \& Rajagopal, K. R. Mathematical issues concerning the Navier-Stokes equations and some of its generalizations. In: Handbook of Differential Equations: Evolutionary Equations, Vol. II, Elsevier/North-Holland, Amsterdam (2005), 371-459. Zbl 1095.35027 MR 2182831

26. Miranville, A., \& Zelik, S. Attractors for dissipative partial differential equations in bounded and unbounded domains. In: Handbook of Differential Equations: Evolutionary Equations, Vol. IV, Elsevier/North-Holland, Amsterdam (2008), 103-200. Zbl 1221.37158 MR 2508165

27. Renardy, M., \& Rogers, R. C. An Introduction to Partial Differential Equations. Texts Appl. Math. 13, Springer, New York (1993). Zbl 0917.35001 MR 1211418

28. SHEn, J., \& YANG, X. Energy stable schemes for Cahn-Hilliard phase-field model of two-phase incompressible flows. Chinese Ann. Math. Ser. B 31 (2010), 743-758. Zbl pre05816628 MR 2726065

29. Siggia, E. D. Late stages of spinodal decomposition in binary mixtures. Phys. Rev. A 20 (1979), 595605.

30. Smagorinsky, J. S. General circulation experiments with the primitive equations. I. The basic experiment. Mon. Weather Rev. 91 (1963), 9-164.

31. Starovoitov, V. N. The dynamics of a two-component fluid in the presence of capillary forces. Math. Notes 62 (1997), 244-254. Zbl 0921.35134 MR 1619861

32. Temam, R. Navier-Stokes Equations. Theory and Numerical Analysis. Reprint of the 1984 edition, AMS Chelsea Publ., Providence, RI (2001). Zbl 0981.35001 MR 1846644

33. ZhaO, L., WU, H., \& HUANG, H. Convergence to equilibrium for a phase-field model for the mixture of two viscous incompressible fluids. Comm. Math. Sci. 7 (2009), 939-962. Zbl 1183.35224 MR 2604626 